\title{
Thermal noise of folding mirrors
}

\author{
D. Heinert, ${ }^{1, *}$ K. Craig, ${ }^{2}$ H. Grote ${ }^{3,4}$ S. Hild, ${ }^{2}$ H. Lück, ${ }^{3,4}$ R. Nawrodt ${ }^{1}$ D. A. Simakov, ${ }^{3,4}$ \\ D. V. Vasilyev, ${ }^{3,5}$ S. P. Vyatchanin, ${ }^{6}$ and H. Wittel ${ }^{3,4}$ \\ ${ }^{1}$ Institut für Festkörperphysik, Friedrich-Schiller-Universität Jena, D-07743 Jena, Germany \\ ${ }^{2}$ SUPA, School of Physics and Astronomy, Institute for Gravitational Research, \\ University of Glasgow, Glasgow G12 8QQ, United Kingdom \\ ${ }^{3}$ Albert-Einstein-Institut, Max-Planck-Institut für Gravitationsphysik, D-30167 Hannover, Germany \\ ${ }^{4}$ Institut für Gravitationsphysik, Leibniz. Universität Hannover, D-30167 Hannover, Germany \\ ${ }^{5}$ Institut für Theoretische Physik, Leibniz Universität Hannover, D-30167 Hannover, Germany \\ ${ }^{6}$ Faculty of Physics, Moscow State University, Moscow 119991, Russia
}

(Received 22 April 2014; published 7 August 2014)

\begin{abstract}
Current gravitational-wave detectors rely on the use of Michelson interferometers. One crucial limitation of their sensitivity is the thermal noise of their optical components. Thus, for example, fluctuational deformations of the mirror surface are probed by a laser beam being reflected from the mirrors at normal incidence. Thermal noise models are well evolved for that case but mainly restricted to single reflections. In this work, we present the effect of two consecutive reflections under a non-normal incidence onto mirror thermal noise. This situation is inherent to detectors using a geometrical folding scheme such as GEO 600 . We revise in detail the conventional direct noise analysis scheme to the situation of non-normal incidence allowing for a modified weighting function of mirror fluctuations. An application of these results to the GEO 600 folding mirror for Brownian, thermoelastic, and thermorefractive noise yields an increase of displacement noise amplitude by $20 \%$ for most noise processes. The amplitude of thermoelastic substrate noise is increased by a factor of 4 due to the modified weighting function. Thus, the consideration of the correct weighting scheme can drastically alter the noise predictions and demands special care in any thermal noise design process.
\end{abstract}

DOI: 10.1103/PhysRevD.90.042001

PACS numbers: 04.80.Nn, 05.40.Ca, 07.60.Ly

\section{INTRODUCTION}

The direct detection of gravitational waves is among the most demanding physical challenges of our time. The ambitious goal of a detection calls for an instrument able to resolve relative length changes in the order of $10^{-21}$ [1]. Interferometric detectors using a Michelson scheme have proven their potential to reach this accuracy in looking for tiny phase differences in their arms. Among them are LIGO [2], Virgo [3], and GEO 600 [4]. While the first two use arm cavities to increase the effect of a gravitational wave onto the detector output, the latter one uses simply folded arms combined with signal recycling [5] in order to achieve a comparable sensitivity.

To reach the sensitivities desired in a gravitational-wave detector, any kind of noise has to be carefully reduced. Thermal noise in particular forms a severe limitation for the detector sensitivity in the most sensitive frequency band from $50 \mathrm{~Hz}$ to $2 \mathrm{kHz}$. In current detectors, the most severe thermal noise process is found in Brownian noise [6]

*daniel.heinert@uni-jena.de

Published by the American Physical Society under the terms of the Creative Commons Attribution 3.0 License. Further distribution of this work must maintain attribution to the author(s) and the published article's title, journal citation, and DOI. emerging due to the thermal motion of the atoms. This leads to a slight deformation of the mirror surfaces changing the arm length, resulting in a signal indistinguishable from the passage of a gravitational wave. If a resonant arm cavity is used in a detector, the phase shift due to a gravitational wave is increased as the light performs an increased number of round trips. At the same time, the effect of a deformed cavity mirror on the laser light is also increased by the number of round trips. For this reason, the noise level of detectors using arm cavities is dominated by the noise of the cavity mirrors. Further, these cavity mirrors are operated at normal incidence.

Using simply folded arms, the situation differs for GEO 600 depicted in Fig. 1. Here, the end test mass (ETM) is probed only once under normal incidence, while the folding mirror $(\mathrm{FM})$ is probed twice, once from the beam entering the arm and once from the beam reflected by the end test mass. Because of the spatial separation of the beams in the interferometer (IFO) arm, the incidence on the folding mirror is not normal. Furthermore, the beam splitter is also probed twice in an interferometric gravitational-wave detector. These two beams cause a standing wave in the beam splitter. Benthem and Levin [7] showed that this standing wave enters the noise calculation of the beam splitter.

In analogy to this idea, the purpose of this paper is to investigate the thermal noise arising from the folding 


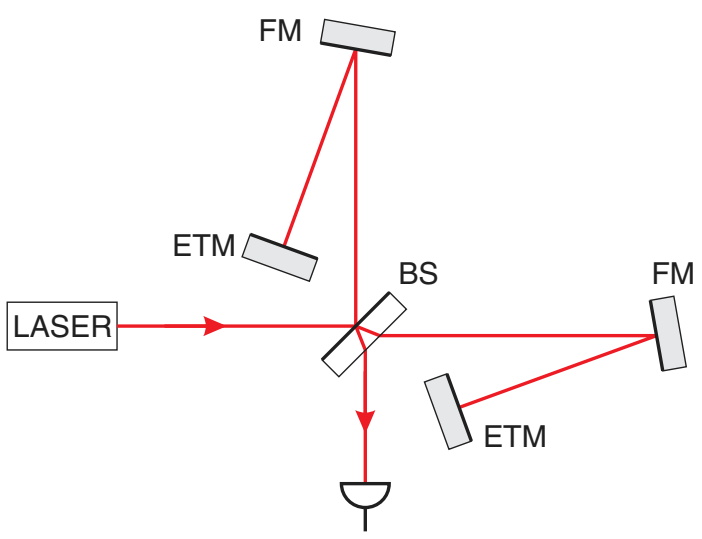

FIG. 1 (color online). Core optics of GEO 600 as a prototype of a gravitational-wave detector with folded arms. Here, the folding mirror is probed twice by the laser light.

mirror. Here, the two incoming beams build up a stripelike intensity pattern suggesting a modification in the noise analysis and a potential change in thermal noise. After repeating the conventional direct approach of noise calculation in Sec. II, we present a qualitative and quantitative justification of a modified noise analysis in Sec. III. Following that, the effects on Brownian, thermoelastic (TE), and thermorefractive (TR) noise for the coating and the substrate are investigated in Sec. IV. Finally, the resulting noise expressions for a striped readout are applied to the GEO 600 geometry and compared to the conventional noise values in Sec. V.

\section{DIRECT NOISE ANALYSIS}

Within this work, we use the direct noise analysis proposed by Levin $[8,9]$. This scheme consists of three steps and is illustrated for Brownian substrate noise in the following. First, the detailed effect of a noisy variable onto the detector's readout has to be evaluated. In this regard, the interferometric detection scheme is sensitive to a phase noise of the light leaving the arm. For the case of Brownian substrate noise, the deformation of the substrate causes such a change in the wavefront of the reflected light. Gillespie and Raab [10] obtained the averaged phase change $\Delta \varphi$ due to this effect as

$$
\Delta \varphi=\frac{4 \pi}{\lambda} \times \int f(x, y) v_{z}(x, y) d x d y,
$$

where $\lambda$ is the wavelength. The surface deformations $v_{z}$ are averaged in the $x-y$ plane via the weighting function

$$
f(x, y)=\frac{1}{\pi r_{0}^{2}} \exp \left(-\frac{x^{2}+y^{2}}{r_{0}^{2}}\right)
$$

representing the intensity distribution of a laser beam with radius $r_{0}$. The phase change $\Delta \varphi$ can be calculated to an effective homogeneous displacement $\bar{v}_{z}$ causing the same effect. This displacement reads

$$
\bar{v}_{z}=\frac{\lambda}{4 \pi} \times \Delta \varphi=\int f(x, y) v_{z}(x, y) d x d y .
$$

In a second step, a virtual harmonic force has to be applied to the substrate's surface mimicking the weighting distribution $f(x, y)$. This is represented by a virtual pressure on the test mass surface of

$$
p(x, y)=F_{0} f(x, y) \cos (\omega t) .
$$

Here, $\omega$ determines the frequency at which the spectral noise power is to be calculated, and $F_{0}$ represents an arbitrary amplitude. With respect to this virtual load, the dissipated power has to be calculated. In our case of Brownian noise, dissipation arises from structural loss in the substrate and is given by the total elastic energy density in the substrate $w_{\max }$ and the mechanical loss $\phi$ via

$$
P_{\mathrm{diss}}=\omega \int \phi(x, y, z) w_{\max }(x, y, z) d V
$$

where the integration is performed over the substrate volume.

Finally, in a third step, the displacement noise power density can be calculated with

$$
S_{z}(\omega)=\frac{8 k_{B} T_{0}}{\omega^{2}} \frac{P_{\text {diss }}}{F_{0}^{2}}
$$

where $k_{B}$ refers to Boltzmann's constant and $T_{0}$ to the temperature.

The direct approach of thermal noise calculation is similar for TE noise. Here, the thermoelastic damping, i.e., a heat flow between regions being expanded and compressed due to the virtual load, represents the dissipation process [11]. In the case of TR noise, instead of a virtual pressure, a virtual entropy enters the test mass, and in analogy to TE noise, the heat flow within the test mass determines the dissipation and, consequently, the TR noise level [9].

\section{WEIGHTING SCHEME FOR A FOLDING MIRROR}

A reliable thermal noise prediction for a folding mirror is preceded by a detailed knowledge of its interaction with the laser beam. As shown in the last section, the direct noise analysis for a simple end test mass exhibiting only a single reflection calls for a virtual load characterized by a Gaussian distribution. Thus, the intensity of the incoming light seems to determine the correct weighting factor $f(x, y)$ for a noise analysis. 


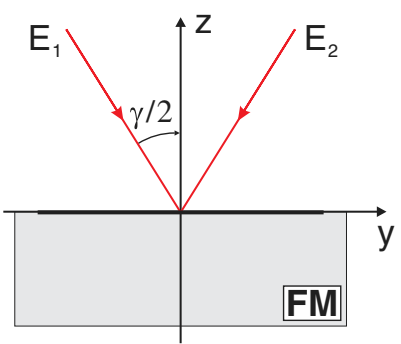

FIG. 2 (color online). Geometric path of the laser beams at the folding mirror surface. Here, we present the incoming light beams $E_{1}$ and $E_{2}$ as well as the coordinate system used for further calculations.

Adapting this rule for the end test mass to the case of a folding mirror calls for the determination of the intensity at the folding mirror surface due to the two incoming waves. Introducing the coordinate system shown in Fig. 2 and assuming plane waves $E_{1}$ and $E_{2}$ (both of amplitude $\hat{E}$ ) approaching the folding mirror, this intensity reads

$$
\begin{gathered}
E(y, z)=\hat{E} e^{i\left(-k_{y} y+k_{z} z\right)}+\hat{E} e^{i\left(k_{y} y+k_{z} z\right)}, \\
=2 \hat{E} e^{i k_{z} z} \cos \left(k_{y} y\right), \\
I(y, z=0)=4 \hat{I} \cos ^{2}\left[k_{0} y \sin \left(\frac{\gamma}{2}\right)\right], \\
=2 \hat{I}\left\{1+\cos \left[2 k_{0} y \sin \left(\frac{\gamma}{2}\right)\right]\right\} .
\end{gathered}
$$

Here, $\hat{I}$ represents the intensity of a single plane wave, $k_{0}=2 \pi / \lambda$ represents the vacuum wave number of the laser light with the wavelength $\lambda$, and $\gamma$ represents the angle between the two light paths in the arm.

In the subsequent analysis, the Gaussian beam profile has to be considered instead of a plane wave. Such a treatment demands for a spatial distribution of the term $\hat{I}$ following the laser beam intensity. The observed intensity pattern on the folding mirror surface in GEO 600 is presented in Fig. 3 and confirms the theoretical prediction. Thus, the intensity-based approach results in a striped read out; deformations at the bright stripes are sensed the most, while any deformation at a dark spot has no effect on the reflected light.

But there exists a second approach to this problem. It relies on the assumption that light entering the arm (beam $E_{1}$ ) and light leaving the arm (beam $E_{2}$ ) are independently reflected by the folding mirror. In GEO 600, the travel time of light in the arm $(\tau=2 L / c \approx 4 \mu \mathrm{s})$ is much shorter than the periodic times of its detection band. Thus, the noise imprinted to the reflected light is likely to be correlated for both reflections at the folding mirror leading to a total noise amplitude being twice as large as for a single reflection. In this picture, noise in the interferometer output is caused by

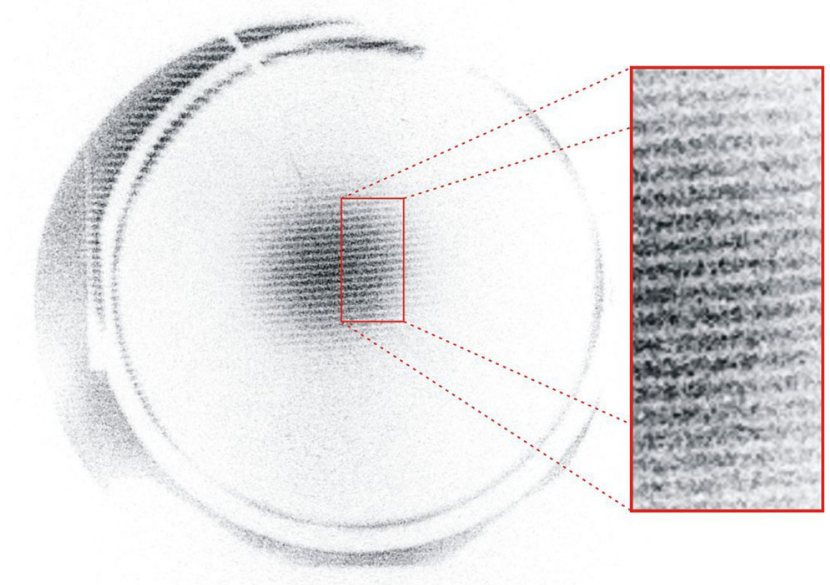

FIG. 3 (color online). Photograph of the intensity pattern at the folding mirror surface in GEO 600. The stripe pattern is clearly visible on top of the Gaussian intensity profile. The stripe period agrees with the theoretical prediction of $\Lambda=2.55 \mathrm{~mm}$. For a better visibility, the intensity of the image has been inverted.

any deformation at the folding mirror surface independently from its position.

At first sight, both approaches seem reasonable but clearly rule out each other. Therefore, in the following parts of this section, we have a closer look into these two models to discover the correct one.

\section{A. Huygen's principle}

A first check is performed by applying Huygen's principle, and for that reason, can be assumed to be quite fundamental. The principle is summarized by two steps. First, an incoming wave excites spherical waves called wavelets at every point on its wavefront. Second, the sum of all wavelets gives the new wavefront of the propagated wave. These two rules allow the construction of the wave reflected by the folding mirror in our case. In Fig. 4, Huygen's principle is applied to a small deformation of the folding mirror surface (referred to as bump). For the following considerations, we assume this bump to possess a dimension being small compared to the intensity period at the folding mirror surface. Further, its height is assumed to be small compared to the laser wavelength.

Applying Huygen's principle to the case of the folding mirror, we begin our discussion at the incoming beam $E_{1}$ from the beam splitter. At the folding mirror, this wave excites mainly wavelets at the undisturbed surface leading to a perfectly reflected plane wave. Only at the bump, roughly speaking, one wavelet is excited being out of phase with the reflected light. This will lead to a very small phase change of the reflected light compared to a perfect reflection. As the deformed area is assumed to be small, no significant amount of energy is scattered out of the reflected light. Thus, in this section, we neglect scattered light and only consider the reflected light in the zeroth order. After 


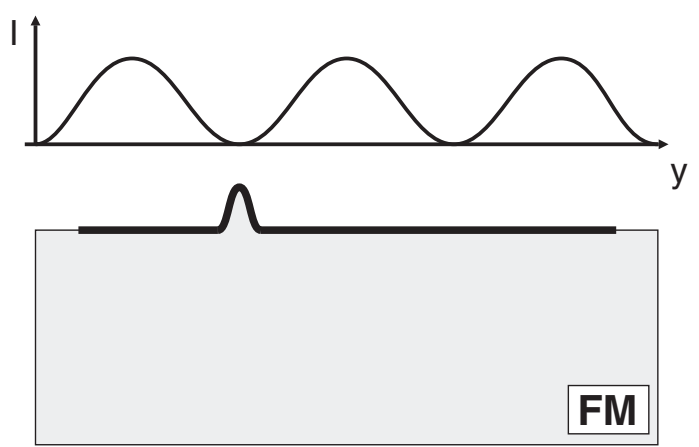

FIG. 4. Sketch of a folding mirror surface deformation to explain its effect on the reflected light using Huygen's principle. The diagram on top shows the intensity distribution at the folding mirror surface. Along the $y$ axis, the deformation is assumed to be small compared to the intensity period along the folding mirror surface and located at minimum intensity. Also, its height is assumed to be small compared to the wavelength.

traveling the long way to the end test mass, we can treat the reflected light to be a perfect plane wave again. This plane wave is then reflected at the end test mass and approaches the folding mirror a second time (as beam $E_{2}$ ). Here, again, mainly a perfectly reflected plane wave is excited. But, again, one wavelet at the deformation shows a different behavior.

Let us assume that the bump is situated at an intensity minimum of the stripe pattern formed by the incoming light $\left(E_{1}\right.$ and $\left.E_{2}\right)$ for the undisturbed case. Even in the disturbed case, the bump will remain in an intensity minimum as the phase change of the plane wave due to the deformation is negligibly small. Clearly, then, at this point, both plane waves $E_{1}$ and $E_{2}$ are out of phase by $180^{\circ}$. Consequently, also, both wavelets excited by the bump show this phase delay and interfere destructively. For this reason, no influence of the bump is expected for the laser light leaving the arm. The situation changes if the bump is situated at an intensity maximum. Then, the two wavelets excited by the bump interfere constructively leading to a maximized signal at the output.

Consequently, Huygen's principle clearly predicts a position-dependent model. In our case, this confirms a weighting by intensity and contradicts the model of independent reflections.

\section{B. Scattering approach}

In a second, more quantitative approach, we discuss the effect of a sinusoidal deformation grating of the folding mirror surface. Here, the deformation solely shows a nonvanishing component along the $z$ direction, following

$$
v_{z}(y)=\hat{v} \sin (K y),
$$

where $\hat{v}$ represents the deformation amplitude and $K=$ $2 \pi / \Lambda$ the wave number of the grating with its spatial period $\Lambda$. As in the case of GEO 600, the incidence is nearly normal $(\gamma \ll 1)$; this surface deformation can be treated as a phase grating exhibiting the following transfer function:

$$
g(y)=\exp \left[i k_{0} \hat{v} \sin (K y)\right] .
$$

Introducing $\Delta \equiv k_{0} \hat{v}$ and assuming a small deformation amplitude $\Delta \ll 1$, the transmitted light of such a phase grating can be approximated as

$$
E_{t}=g(y) E_{i} \approx\left(1+\frac{\Delta}{2} e^{i K y}-\frac{\Delta}{2} e^{-i K y}\right) E_{i} .
$$

Here, $E_{i}$ depicts the incoming electric field, and the terms in brackets can be identified as emerging diffraction orders caused by the grating. The first term describes the unperturbed beam, while the second and third terms represent the first diffraction orders showing a linear amplitude dependence from the deformation amplitude. In our case, we have to cover a reflection grating. This can be approximated by a single transmission through the phase grating, the reflection at the folding mirror coating stack, and a second transmission through the grating. Thus, Eq. (13) remains valid with the replacement $\Delta \rightarrow 2 \Delta$.

Understanding the process of a single reflection on a periodically deformed mirror forms the basis for the noise estimate of a complete IFO arm. We are especially interested in the impact of scattering onto the phase of the light reflected from the arm. In the following, we focus on a linear noise response, i.e., taking all light paths with a single scattering into a nonzero diffraction order of the surface grating into account.

These paths are illustrated in Fig. 5. Note that the scattered beams only remain within the IFO if the diffracted light coincides with the reflected beam, leading to the relation

$$
K=2 k_{y}=2 k_{0} \sin (\gamma / 2) .
$$

This relation shows that only a grating with the same period as the intensity field from Eq. (10) leads to a noise effect in the detector. One can interpret this fact as a first confirmation of the intensity-based readout model.

In a mathematical description of this process, we construct the optical field at the beam splitter as

$$
E_{\text {out }}=e^{i k_{0} L_{0}}\left(\Delta+e^{i 2 k_{0} L}-\Delta e^{i 4 k_{0} L}\right) e^{i k_{0} L_{0}} E_{\text {in }} .
$$

Here, the distance between the beam splitter and the folding mirror is given by $L_{0}$, while the length of the folded arm (FM to ETM) is given by $L$. The first term in brackets originates from the directly backscattered light [Fig. 5(b)], the second term from the unperturbed process [Fig. 5(a)], and the third term from the doubly revolving beam [Fig. 5(c)]. Equation (15) can also be discussed in analogy 


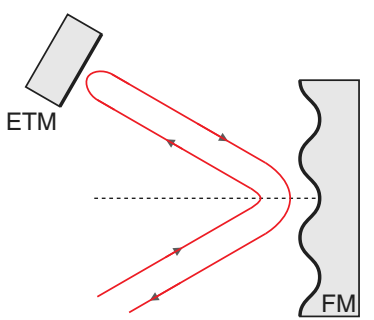

(a)
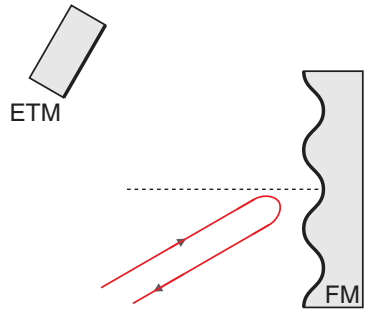

(b)

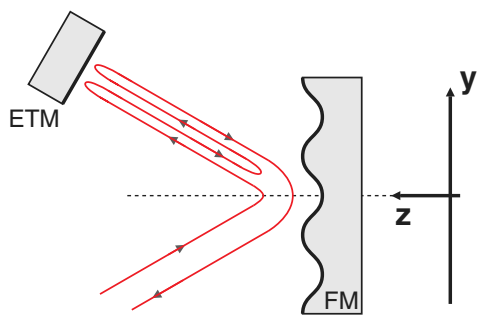

(c)
FIG. 5 (color online). Scatter paths of first order due to a surface grating at the folding mirror. In (a), the unperturbed light path is shown with simple reflections at the folding mirror. Case (b) represents the direct backscatter of the incoming light in the negative diffraction order. This light does not enter the folded arm at all. In (c), the light is reflected at the folding mirror and the ETM and scattered back into the folded arm at its second approach to the folding mirror. This scatter path uses the positive diffraction order and shows a double round trip in the folded arm. The sum of all these paths adds up to the total optical field reflected from the IFO arm in first order.

to a weakly coupled cavity. There in the leading order, the same three light paths exist: a first one directly reflected at the input mirror (without any round trip), a second one showing a single round trip, and a third path exhibiting two round trips in the cavity.

Simplifying Eq. (15) yields

$$
E_{\text {out }}=e^{i 2 k_{0}\left(L_{0}+L\right)}\left[1-2 i \Delta \sin \left(2 k_{0} L\right)\right] E_{\text {in }} .
$$

At first glance, it seems counterintuitive that the phase of the reflected light depends on the length of the folded arm. But a change in arm length $L$ does affect the intensity pattern at the folding mirror surface. At the same time, the surface deformations stay constant as defined in Eq. (11). Consequently, the deformation moves with respect to the intensity pattern and for that reason undergoes a periodic variation following $\sin \left(2 k_{0} L\right)$. Below in our calculations, we consider the maximum impact on the reflected light's phase, i.e., replacing the sine term by 1 . This is justified by the fact that one can always find a surface deformation leading to this maximum signal. Thus, the phase of the reflected light reads

$$
\Delta \varphi=\arg \left(\frac{E_{\text {out }}}{E_{\text {in }}}\right) \approx-2 \Delta=-2 k_{0} \hat{v} .
$$

The last equation gives the foundation of Levin's noise analysis as it links the effect of a surface deformation to a phase change in the light leaving the arm. A homogeneous deformation of the folding mirror surface by $\hat{v}$ is probed by the light from the beam splitter and by the light from the end test mass resulting in a total phase shift of $4 k_{0} \hat{v}$. It tells us that a sinusoidal deformation with amplitude $\hat{v}$ shows half the effect on the reflected light's phase compared to a homogeneous surface deformation of the same amplitude $\hat{v}$.

Summarizing the previous considerations, we find that the laser light reflected from the folded arm is only sensitive to a homogeneous deformation or a spatial grating with wave number $K \approx k_{0} \gamma$. In the above result, the approximation of small tilting angles $\gamma$ has been used. The diffracted orders of gratings with another spatial frequency will be scattered out of the beam path and consequently do not cause a signal on the detector output. Thus, the averaged readout variable in terms of an effective displacement follows from Eq. (3) using a weighting function of

$$
f_{\mathrm{FM}}(x, y)=C \exp \left(-\frac{x^{2}+y^{2}}{r_{0}^{2}}\right)\left[1+\cos \left(k_{s} y\right)\right]
$$

where the wave number of the grating $k_{s}=2 \pi / \Lambda \approx k_{0} \gamma$ and a constant $C$ have been introduced. For calculational reasons, the sine function has been replaced by a cosine function being equivalent to a length change of the arm. The effect of this replacement will be negligibly small for our case of interest, i.e., small stripes compared to the beam radius. In this case, we also find that the averaged displacement of a homogeneous deformation is twice as high as for the sinusoidal deformation of the surface in agreement with the previous considerations. Thus, the presented scatter approach demands the application of an intensity weighted probe force.

\section{THERMAL NOISE CALCULATION}

In the last section, it turned out that in a direct noise analysis scheme following Levin [8] an intensity weighted probe load has to be applied to the folding mirror. This load type differs from the conventional model for a single incidence on a mirror. For that reason, we want to derive and discuss the deviations from the conventional models in this section. Within this calculation, we restrict ourselves to an analysis of Brownian, TE, and TR noise. As the folding mirror represents a fully reflective component, we only consider TR noise in the coating. For the sake of simplicity, we do not perform a thermo-optic noise analysis as a coherent modelling of TE and TR noise. Rather, we treat both mechanisms independently. 
TABLE I. Geometrical and optical properties of GEO 600's folding mirror. The number of dielectric $\lambda / 4$ layer pairs has been estimated by the measured transmittance of $\simeq 10 \mathrm{ppm}$.

\begin{tabular}{ll}
\hline \hline Substrate & \\
Diameter $2 R$ & $18 \mathrm{~cm}$ \\
Height $H$ & $10 \mathrm{~cm}$ \\
Temperature $T_{0}$ & $300 \mathrm{~K}$ \\
Material & Fused silica \\
Coating & \\
$\#$ of $\lambda / 4$ layers & \\
Tantala & 20 \\
Silica & 20 \\
Laser beam $\sqrt{2} r_{0}$ & \\
Radius $w=\sqrt{ }$ & \\
Wavelength $\lambda$ & $25 \mathrm{~mm}$ \\
Stripe period $\Lambda$ & $1064 \mathrm{~nm}$ \\
Folding angle $\gamma$ & $2.55 \mathrm{~mm}$ \\
\hline \hline
\end{tabular}

\section{A. Probing load}

For an analysis of Brownian and TE noise, a virtual force has to be applied to the folding mirror surface. This force results from the averaging to a total effective displacement $\bar{v}_{z}$ using

$$
\bar{v}_{z}=\int f_{\mathrm{FM}}(x, y) v_{z}(x, y) d x d y
$$

using the weighting function $f_{\mathrm{FM}}(x, y)$ given in Eq. (18). In this expression, near to normal incidence has been assumed as otherwise an elliptical beam shape has to be taken into account. A homogeneous deformation $v_{z}(x, y)=v_{0}$ should result in the same value for the effective displacement $\bar{v}_{z}=v_{0}$ and thus determines $C$ from Eq. (18) as

$$
C=\frac{1}{\pi r_{0}^{2}} \frac{1}{e^{-k_{s}^{2} r_{0}^{2} / 4}+1} .
$$

In this work, we focus on the limit of small stripe periods compared to the laser beam radius $\left(k_{s} r_{0} \gg 1\right)$, which is clearly valid for GEO 600 (see Table I). This fact leads to a simplification of $C$ and finally to a virtual pressure of

$$
p(x, y)=\frac{F_{0}}{\pi r_{0}^{2}} \exp \left(-\frac{x^{2}+y^{2}}{r_{0}^{2}}\right)\left[1+\cos \left(k_{s} y\right)\right],
$$

which has to be applied to the folding mirror surface.

\section{B. Brownian coating noise}

We choose Brownian coating noise to intuitively illustrate a noise increase due to a striped readout. In our simplified model, the coating is assumed to be linearly coupled only to the substrate and not to its neighboring coating regions. These conditions can be modeled by

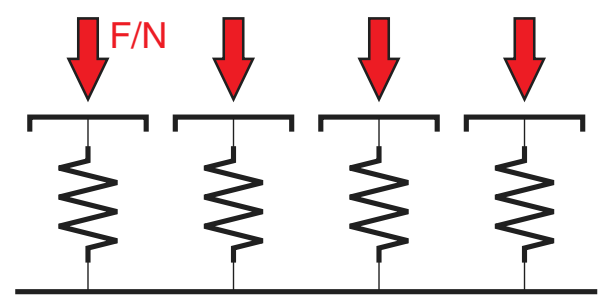

(a) homogeneous load

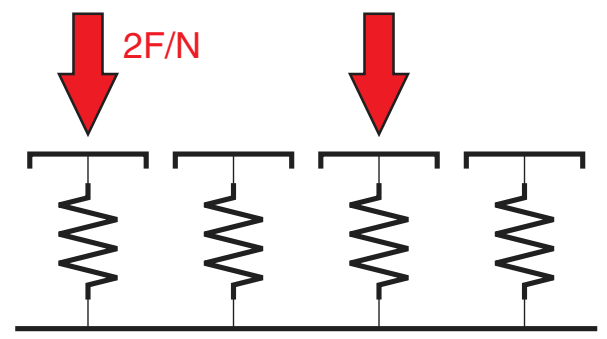

(b) striped load

FIG. 6 (color online). Model system of a coating layer on top of a substrate. In this model, no interaction within the layer and a linear coupling to the substrate is assumed. In scheme (a), the coating is probed by a homogeneous readout, while in (b), a striped readout is illustrated.

dividing the coating into little domains. Each domain is separately coupled to the substrate and modelled as a spring. This geometry is presented in Fig. 6. In a first step, we calculate the energy stored in the springs representing the energy stored in the coating for a homogeneous load. Considering $N$ sections with a total force $F_{0}$, we find

$$
E_{\mathrm{hom}}=N \frac{\left(F_{0} / N\right)^{2}}{2 S},
$$

with $S$ representing the spring constant of a single spring. To model an inhomogeneous load scheme, we only apply forces to every second domain. Thus, the force on each loaded domain is doubled, but the number of loaded domains is halved. The energy in the coating then reads

$$
E_{\text {stripe }}=\frac{N}{2} \frac{\left(2 F_{0} / N\right)^{2}}{2 S}=2 E_{\mathrm{hom}},
$$

leading to a doubled amount of energy stored in the coating. This result would also cause a doubling of the noise power density. A final and more realistic approach has been done for a sinusoidal load distribution. Without showing the calculations for this case, an energy increase of 50\% compared to the homogeneous case has been observed.

A detailed analytical calculation of Brownian coating noise is presented in Appendix E. This approach assuming a half-space substrate totally confirms our rough model and exactly shows an increase by $50 \%$ in coating energy and thus in Brownian coating noise power due to the emergence of stripes. Thus, for a semi-infinite substrate and a striped readout, Brownian coating noise reads 


$$
S_{\mathrm{Br}, \text { coat }}=\frac{3}{2} S_{\mathrm{Br}, \text { coat }}^{\text {(conv }},
$$

where the conventional term follows the result of Harry et al. [12].

Further, a finite element analysis has been performed to estimate the change due to the finite size of substrates. For the geometry of GEO 600's substrate, this revealed reduced noise levels with small corrections below $10 \%$.

\section{Brownian substrate noise}

To take a striped readout into account in the noise power density of Brownian substrate noise, one can introduce a correction function $G$ :

$$
S_{\mathrm{Br}, \text { sub }}=G\left(\frac{k_{s} r_{0}}{\sqrt{2}}\right) S_{\mathrm{Br}, \text { sub }}^{\text {(conv) }}
$$

Here, the conventional term for the half-space is given by the work of Bondu, Hello, and Vinet [13]. In the limit of small stripes compared to the laser beam, the correction factor reads

$$
\begin{aligned}
G(b)= & +2 e^{-3 b^{2} / 8} I_{0}\left(b^{2} / 8\right)+\frac{1}{2} e^{-b^{2}} \\
& +\frac{1}{2} e^{-b^{2} / 2} I_{0}\left(b^{2} / 2\right),
\end{aligned}
$$

where $I_{0}$ represents the modified Bessel function of the first kind. For a sketch of derivation of this expression, see Appendix A. Using GEO 600 parameters reveals a value of $b \approx 45$, leading to a correction factor of $G(b) \approx 1.0063$. Thus, with an increase of $0.3 \%$ in the noise amplitude, the effect of stripes on Brownian substrate noise is negligibly small in GEO 600.

\section{Thermoelastic substrate noise}

In the direct calculation of TE noise, the virtually applied surface load leads to a volume dilatation in the substrate. Consequently, heat is produced in these dilated regions, and a heat flow sets in representing the dissipative process. In contrast to the conventional Gaussian load, a striped readout reduces the separation between dilated and nondilated regions. The heat flow between these regions is increased, suggesting also an increased TE noise. This effect has been analytically investigated for LaguerreGauss modes of higher order by Vinet [14]. Thus, also in our case, the evaluation of TE substrate noise might be drastically increased, demanding a careful examination.

A detailed analysis of this problem is presented in Appendix B. With the assumptions of small stripes compared to the laser beam $\left(k_{s} r_{0} \gg 1\right)$ and a small heat propagation length compared to the beam radius $\left[\kappa /\left(\rho C_{p} \omega\right) \ll r_{0}\right]$, we find a noise spectrum:

$$
S_{\mathrm{TE}, \mathrm{sub}}=\frac{8}{\sqrt{2 \pi}} \frac{\kappa \alpha^{2}(1+\sigma)^{2}}{\rho^{2} C_{p}^{2}} \frac{k_{B} T_{0}^{2}}{r_{0}^{3}} \frac{1}{\omega^{2}}\left(1+\frac{k_{s} r_{0}}{\sqrt{2 \pi}}\right) \text {. }
$$

Here, the first term coincides with the conventional solution by Braginsky et al. [11], while the second term is due to the stripe's influence and, especially for small stripe periods, can be significantly larger than the conventional term. An estimate of the maximum noise enhancement at $100 \mathrm{~Hz}$ is presented below. As Eq. (27) is only valid for small thermal path lengths $\left(k_{s}^{2} a^{2} \ll \omega\right)$, a maximum value of

$$
k_{s}=\sqrt{\frac{\omega}{a^{2}}} \approx 27.3 \times 10^{3} \mathrm{~m}^{-1}
$$

is obtained. The above value corresponds to a stripe period of $230 \mu \mathrm{m}$. Inserting this number into the noise term due to stripes as given in Eq. (27) reveals a maximum increase in TE noise power by a factor of $\sim 200$.

Using the more profound Eq. (B15) from Appendix B, the influence of the stripe period $\Lambda$ on the TE substrate noise is illustrated in Fig. 7. In GEO 600, the stripe period $\Lambda=2.55 \mathrm{~mm}$ is rather large. This leads to a $1 / \omega$ decrease of the TE noise amplitude above $2 \mathrm{~Hz}$ and justifies the use of Eq. (27) as an approximation toward high frequencies. At low frequencies, the approximation of an adiabatic heat flow between the stripes is violated, resulting in a constant TE noise level. At even lower frequencies, the TE noise amplitude passes over to the conventional curve, showing again a $1 / \omega$ dependency.

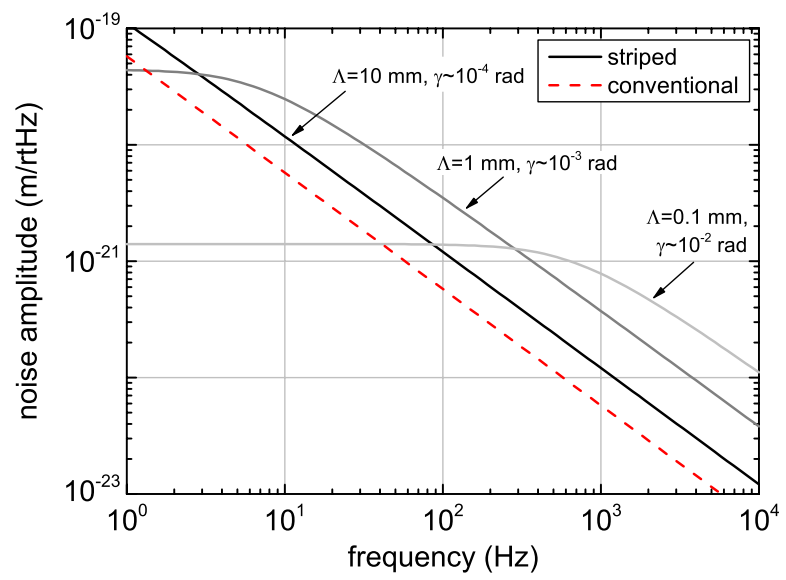

FIG. 7 (color online). Thermoelastic noise amplitude for a striped readout and different stripe periods $\Lambda$. In analogy to substrate TR noise in a beam splitter [7], a region of constant noise emerges at low frequencies. Its cutoff frequency increases by decreasing the stripe period. The dashed red curve marks the conventional noise contribution. It has to be independently added to the stripe noise level to obtain the total TE noise. For relatively large stripe periods, a significant increase in noise can be found within the typical frequency band of gravitational-wave detectors. At high frequencies, the $1 / \omega^{2}$ dependence from Eq. (27) is visible. 


\section{E. Thermoelastic coating noise}

Besides the substrate, also the coating introduces TE noise. In a direct noise analysis, the virtual force results in a volume dilatation of the coating. Via thermal expansion, this leads to the emergence of heat sources in the coating. In our analysis, we model these heat sources to be infinitely thin. Thus, any heat flow within the coating is neglected. In GEO 600, the thermal path length in the substrate (fused silica) is small compared to the stripe period, and the stripe period is small compared to the beam radius. These assumptions lead to a TE coating noise of

$$
S_{\mathrm{TE}, \mathrm{coat}}=\frac{\sqrt{2}}{\pi} \frac{\hat{\alpha}^{2} Y_{c}^{2} M^{2}}{\sqrt{\kappa \rho C_{p}}} k_{B} T_{0}^{2} \frac{H^{2}}{r_{0}^{2}} \frac{1}{\sqrt{\omega}}\left(1+\frac{1}{2}\right),
$$

where $\hat{\alpha}$ is the difference in the coefficient of thermal expansion between the coating and the substrate and $H$ the coating thickness. $M$ contains the elastic properties of the coating and the substrate via

$$
M=\frac{1}{1-\sigma_{c}}\left[-\frac{1+\sigma_{c}}{Y_{c}}+\frac{1+\sigma}{Y}(2 \sigma-1)\right],
$$

where $Y$ and $\sigma$ represent the substrate's Young's modulus and Poisson ratio, respectively, while the index $c$ refers to the respective coating properties. A detailed derivation of TE coating noise is given in Appendix C. The first term in brackets of Eq. (29) marks the solution of the conventional case coinciding with the former result of Braginsky and Vyatchanin [15]. The second term represents the increase due to the striped readout. There, an increase by $50 \%$ in noise power due to the change to the striped readout pattern is visible.

The presented expression for a single coating layer can be adapted to a high reflective multilayer coating by independently adding the heat sources in the heat equation [see Eq. (B1)]. Consequently, the terms $\alpha M H$ have to be added for every single layer, and finally this sum has to be inserted into Eq. (29). This approach remains valid for small stacks as long as the thermal path length in the coating is larger than the coating height.

In GEO 600 at $10 \mathrm{kHz}$ the thermal path length approaches the thickness of the coating stack. Then, also, a heat flow within the layers probing their thermal properties has to be taken into account. As this has not been done in our analysis, the results above frequencies of $1 \mathrm{kHz}$ are brave extrapolations and should be handled with care. The same arguments remain valid for the TR coating noise presented in the next subsection.

\section{F. Thermorefractive coating noise}

Thermorefractive noise is caused by temperature fluctuations in the coating and an accompanying change in refractive index $n$ mediated by the thermo-optic coefficient $\beta=d n / d T$. For a thick classical $\lambda / 4$ stack and a homogeneous temperature distribution, an effective coefficient $\beta_{\text {eff }}$ can be obtained $[16,17]$ as

$$
\beta_{\mathrm{eff}} \equiv \frac{n_{L}^{2} B_{H}+n_{H}^{2} B_{L}}{4\left(n_{H}^{2}-n_{L}^{2}\right)} .
$$

In the equation above, the indices $L$ and $H$ refer to the low and high index material, respectively. For the pure TR effect, $B_{i}$ is given by the thermo-optic coefficients $\beta_{i}$ of each layer.

Further, the considered temperature change leads to a variation in the geometrical height of the coating layers and enters the reflection characteristics of the stack. This effect has not been considered in the TE coating noise calculation and has to be added to the TR coating noise. Following the suggestion by Fejer et al. [18], this is realized by replacing the coefficient $B_{i}$ by

$$
B_{i}=\beta_{i}+n_{i} \bar{\alpha}_{i},
$$

with

$$
\bar{\alpha}_{i} \equiv \frac{1+\sigma_{i}}{1-\sigma_{i}} \alpha_{i},
$$

using Poisson ratio $\sigma$, the coefficient of linear thermal expansion $\alpha$, and the indices $i=L, H$.

A detailed calculation sketched in Appendix D gives the spectral noise density of TR coating noise. In the approximation of a stripe period large compared to the thermal path length, it yields

$$
S_{\mathrm{TR}, \text { coat }}=\frac{\sqrt{2}}{\pi} \frac{\beta_{\mathrm{eff}}^{2}}{\sqrt{\kappa \rho C_{p}}} k_{B} T_{0}^{2} \frac{\lambda^{2}}{r_{0}^{2}} \frac{1}{\sqrt{\omega}}\left(1+\frac{1}{2}\right),
$$

using the laser wavelength $\lambda$. Again, the first term in brackets represents the original result, while the second term emerges from the stripe readout.

\section{NOISE RESULTS FOR THE FOLDING MIRROR OF GEO 600}

In this section, all the presented noise formulas are applied to the folding mirror of GEO 600. Its geometrical and optical properties are summarized in Table I. Further, the material parameters underlying the numerical results are presented in Table II.

With this information, we first check the assumptions made in the derivation of the noise equations. The first assumption was on the laser beam being large compared to the stripe pattern's period. In an analytical way, it reads

$$
k_{s}^{2} r_{0}^{2} \approx 1900 \gg 1
$$

and is well fulfilled for GEO 600. Especially for TE and TR noise, several assumptions on the thermal path length are 
TABLE II. Material properties of GEO 600's substrate and coating materials at $300 \mathrm{~K}$. Except where noted, the parameters have been taken from Ref. [18]. Because of a lack of better knowledge, most of the silica coating properties are adopted from the bulk fused silica. The mechanical loss of the fused silica substrate is oriented on measured values. Thus, in this work, we do not use an extrapolation to bulk losses as proposed by Penn et al. [19].

\begin{tabular}{lccc}
\hline \hline & Fused silica (substrate) & Silica (coating) & Tantala \\
\hline Young's modulus $Y[\mathrm{GPa}]$ & 72 & 72 & 140 \\
Poisson ratio $\sigma$ & 0.17 & 0.17 & 2200 \\
Mass density $\rho\left[\mathrm{kg} \mathrm{m}^{-3}\right]$ & 2200 & $5 \times 10^{-5}[20]$ & 0.23 \\
Mechanical $\operatorname{loss} \phi$ & $1 \times 10^{-8}$ & 746 & $2.4 \times 10^{-4}[21]$ \\
\hline Specific heat $C_{p}\left[\mathrm{~J} \mathrm{~kg}^{-1} \mathrm{~K}^{-1}\right]$ & 746 & $0.5[22]$ & 306 \\
Thermal conductivity $\kappa\left[\mathrm{W} \mathrm{m}^{-1} \mathrm{~K}^{-1}\right]$ & 1.38 & $5.1 \times 10^{-7}$ & $0.6[22]$ \\
Thermal expansion $\alpha\left[\mathrm{K}^{-1}\right]$ & $5.1 \times 10^{-7}$ & 1.45 & $3.6 \times 10^{-6}$ \\
\hline Refractive index $n$ & $1.45[7]$ & $8.5 \times 10^{-6}$ & 2.03 \\
Thermo-optic coefficient $\beta\left[\mathrm{K}^{-1}\right]$ & $8.5 \times 10^{-6}[7]$ & $14 \times 10^{-6}[17]$ \\
\hline \hline
\end{tabular}

needed. At an exemplary frequency of $100 \mathrm{~Hz}$ we obtain for fused silica

$$
r_{\mathrm{th}}=\sqrt{\frac{a^{2}}{\omega}}=\sqrt{\frac{\kappa}{\rho C_{p} \omega}}=37 \mu \mathrm{m} .
$$

For the conventional results, it is assumed that the beam radius is large compared to the thermal path length. Mathematically, we obtain

$$
\left(r_{\text {th }} \frac{6}{r_{0}}\right)^{4} \approx 2.5 \times 10^{-8} \ll 1,
$$

promising the reliability of the approximation. A helpful approximation of the final result for coating TE and TR noise uses the assumption of a thermal path length being small compared to the stripe period. Even this most restrictive approximation is justified in GEO 600 via

$$
\left(r_{\text {th }} \frac{2 \pi}{\Lambda}\right)^{2} \approx 6.9 \times 10^{-5} \ll 1
$$

The results of the noise analysis for GEO 600's folding mirror are presented in Fig. 8, where they are compared to the conventional theory without stripes. For the conventional estimate, we used Ref. [23] for substrate TE and Brownian noise and Ref. [24] for coating Brownian noise. All of these references include the effects due to a finite substrate size. Further, for coating TE (Ref. [15]) and coating TR (Ref. [16]) noise, we used models not considering a finite-sized substrate but a half-space. This is motivated by the fact that the error in coating noise due to the substrate's finite size is moderately low for the geometry of GEO 600 mirrors (see Ref. [24]).

In the conventional case, the Brownian coating noise dominates the noise spectrum followed by Brownian substrate noise for which the amplitude is roughly half as large. At frequencies above $1 \mathrm{kHz}$ TE coating noise can become important. But taking the coherent effect of TE and TR coating noise into account $[17,25]$ reduces their total noise level.

Turning to the striped case, an increase in the noise amplitude by $22 \%$ for most noise processes has been found. Among them is also coating Brownian noise that still dominates the mirror's noise. In contrast, substrate Brownian noise stays the same as in the conventional case. This has been analytically confirmed for the half-space, but finite element calculations also confirmed this result for GEO 600's finite geometry. Further, substrate TE noise is strongly increased by roughly a factor of 4 . This would lead to a strong influence at frequencies below $3 \mathrm{~Hz}$; however, this frequency range is not crucial for GEO 600's detection band. Summing up, the total noise amplitude is increased by a $22 \%$ in GEO 600's detection band if the striped readout is taken into account.

In a final effort, the resulting noise by GEO 600's folding mirror is to be compared to the measured sensitivity curve

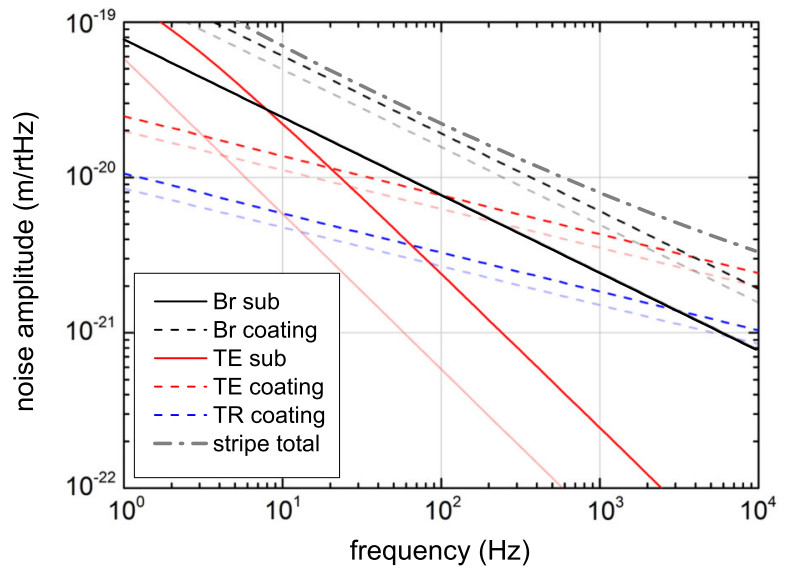

FIG. 8 (color online). Displacement noise of GEO 600's folding mirror. The diagram shows the noise levels for the striped configuration as opaque lines. For comparison, the results of the conventional case have been added as transparent curves. Further, the substrate contributions are solid lines, while the coating contributions appear dashed. 
in terms of the gravitational wave strain $h$. For this purpose, we have to compare the effect of a gravitational wave of amplitude $h$ with the effect of a folding mirror displacement $\Delta z$ to the output of the interferometer. For a simple Michelson interferometer, a gravitational wave of amplitude $h$ leads to a phase shift of [1]

$$
\Delta \varphi=\frac{4 \pi}{\lambda} h L_{\mathrm{tot}},
$$

between the arms. Here, the unfolded arm length, i.e., $L_{\text {tot }}=2 L=1200 \mathrm{~m}$ for GEO 600 , has to be inserted. Further, a homogeneous displacement $\Delta z$ of the surface of one of the folding mirrors leads to a phase change

$$
\Delta \varphi=\frac{4 \pi}{\lambda} 2 \Delta z .
$$

The additional factor of 2 arises due to the occurrence of two reflections on the folding mirror. This doubling of the noise amplitude represents a full correlation of the deformations between the two reflections at the folding mirror. In GEO 600, this correlation is justified as the travel time of light in GEO 600's arm is short compared to the interesting frequencies in the detector. Using Eqs. (39) and (40), a conversion from $\Delta z$ to $h$ is obtainable implying a calculational rule for the GW strain noise density as

$$
S_{h}(\omega)=\frac{8}{L_{\mathrm{tot}}^{2}} S_{z}(\omega) .
$$

In the above expression, an additional factor of 2 has been included resulting from the folding mirror of the second arm. This second folding mirror also produces noise being uncorrelated with respect to the first folding mirror and thus is simply to be added in terms of noise power. The resulting spectrum in terms of noise amplitude is presented in Fig. 9.

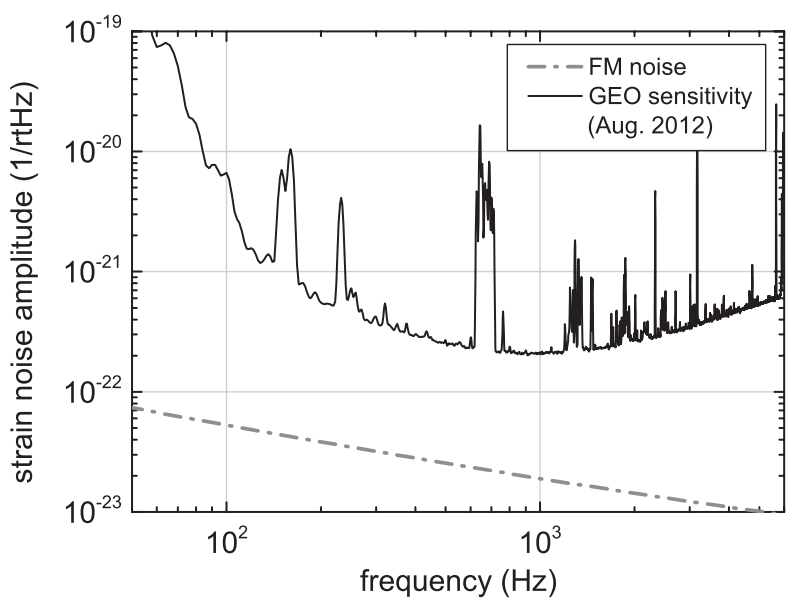

FIG. 9. Thermal strain noise $S_{h}$ of the folding mirror in GEO 600 compared to the measured sensitivity curve. The latter has been taken from Ref. [26]. Thermal noise of the folding mirror is not to limit the current sensitivity of GEO 600 .
A comparison to the measured sensitivity reveals that the folding mirror thermal noise is at least a factor of 10 below the measured data. Consequently, the current sensitivity is not limited by thermal noise of the folding mirror even taking the effect of a striped readout into account.

Within this work, the intensity pattern has been assumed to show a constant spatial distribution. A closer investigation of GEO 600's locking scheme actually reveals that the intensity on the folding mirror surface is moving over the sample surface. This effect corresponds to the motion of the folding mirror for which the position is not controlled in the locking scheme of GEO 600. By a direct inspection of the folding mirror, this motion is experimentally observed (see Fig. 3). Its amplitude turns out to be well below five stripe periods at the folding mirror surface. Further, it occurs at the folding mirror fundamental pendulum mode near $1 \mathrm{~Hz}$.

As this frequency turns out to be well below the desired detection band, we did not consider this effect in our analysis. Further, a translational motion of the readout variable represents a transient load case and is not included in the current direct approach of the fluctuation-dissipation theorem. For this reason, we leave the necessary implementation of nonstationarity and the calculation of detailed results for future work.

\section{CONCLUSION}

In the field of gravitational-wave detection, thermal noise analyses are mainly restricted to single reflections of a laser beam on a mirror at normal incidence. In this work, we covered the case of GEO 600's folding mirror that is probed twice and away from normal incidence. Two possible ways of noise evaluation have been discussed, and the weighting by the intensity profile has been identified to be correct. In this sense, our work agrees with the idea behind the noise calculation in the beam splitter by Benthem and Levin [7]. Nevertheless, a general theorem combining the intensity pattern and the readout profile for a noise analysis is still missing. This would be helpful for future noise estimates not only in the field of gravitational-wave detection. It would further confirm the application of an intensity weighted load in a direct noise calculation scheme. Such a scheme is widely used in the calculation of thermal noise in whispering gallery resonators $[27,28]$ to obtain a limit on their frequency stability. As our work expands the validity of an intensity weighting to the case of a folding mirror, it strengthens its validity.

We worked out the noise expression considering the striped readout scheme for GEO 600. An evaluation of the noise terms revealed that the measured noise in GEO 600 is well above the predicted thermal noise. Thus, the current sensitivity is not limited by the folding mirror thermal noise. But, especially, TE substrate noise shows a large dependency of the stripe period. It is thus suited to adjust the TE noise level without changing the sample in a direct 
noise measurement. This method will increase the reliability of such a measurement.

Recently, the use of folded cavities was proposed by Ballmer and Ottaway [29]. It promises a reduction of coating Brownian noise as the limiting thermal noise contribution in current detectors. For this approach, a single beam is repeatedly reflected on the same mirror but on spatially separated spots of each mirror. Such a scheme leads to an effective increase of the probed mirror surface promising the mentioned noise reduction. Our results also affect this idea at least for a standing wave configuration. The emergence of a striped readout pattern will thus decrease the possible benefit in detector sensitivity using folded cavities.

Finally, in this work, we omitted to investigate the effect of stripes on the thermal noise of GEO 600's beam splitter. As GEO 600 does not exhibit arm resonators, the beam splitter noise in contrast to other detectors is not suppressed at the output. Thus, a detailed investigation of the beam splitter noise also in terms of stripes remains an interesting topic for future work.

\section{ACKNOWLEDGMENTS}

For their constructive questions and fruitful discussions, we thank F. Khalili, J. Hough, K. Somiya, B. Willke, and W. Kells. Further, we thank I. Martin for his helpful comments on the manuscript. D. H. and R. N. acknowledge the support of the German Science Foundation (DFG) under Contract No. SFB Transregio 7. S. P. V. is supported by the Russian Foundation for Basic Research, Grants No. 14-02-00399 and No. 13-02-92441 (ASPERA), and NSF Grant No. PHY-130586. S. H. is grateful for the support from the European Research Council (Grant No. ERC-2012-StG: 307245). D. V. V. acknowledges support from the Centre for Quantum Engineering and Space-Time Research (QUEST) at the Leibniz Universität Hannover and funding by the Vienna Science and Technology Fund (WWTF) through Project No. ICT12049. D. A. S. and H. W. are grateful for the support from the Max-Planck society and from the international Max Planck Research School (IMPRS). Parts of us are further supported by the Science and Technology Facilities Council (STFC) through Grant No. ST/L000946/1, the International Max Planck Partnership, and by the ASPERA ET-R\&D project.

\section{APPENDIX A: BROWNIAN SUBSTRATE NOISE}

In the direct application of the fluctuation-dissipation theorem, the elastic reaction of the substrate on the surface load,

$$
p(x, y)=\frac{F_{0} \cos (\omega t)}{\pi r_{0}^{2}} \exp \left(-\frac{x^{2}+y^{2}}{r_{0}^{2}}\right)\left[1+\cos \left(k_{s} y\right)\right],
$$

has to be revealed. The dissipated power in this case is caused by structural damping in the substrate and follows
$P_{\text {diss }}=\omega \phi E$, where $\phi$ represents the mechanical loss of the substrate and $E$ the maximum elastic energy stored in the substrate. The main part of this appendix is on the calculation of the elastic energy stored in the substrate.

In our calculation, we focus on the response of a semiinfinite test mass, thus neglecting all deviations due to a finite geometry. For this assumption, the deformation in the test mass is applicable by a Green's function approach (see. Ref. [30]). For the case of a pure surface load along the $z$ direction, it reads

$$
\begin{aligned}
v_{x}(x, y, z)= & \frac{1+\sigma}{2 \pi Y} \\
& \times \int d x^{\prime} d y^{\prime}\left(x-x^{\prime}\right)\left(\frac{z}{r^{3}}-\frac{1-2 \sigma}{r(r+z)}\right) f_{z}\left(x^{\prime}, y^{\prime}\right),
\end{aligned}
$$

$$
\begin{aligned}
v_{y}(x, y, z)= & \frac{1+\sigma}{2 \pi Y} \\
& \times \int d x^{\prime} d y^{\prime}\left(y-y^{\prime}\right)\left(\frac{z}{r^{3}}-\frac{1-2 \sigma}{r(r+z)}\right) f_{z}\left(x^{\prime}, y^{\prime}\right),
\end{aligned}
$$

$$
v_{z}(x, y, z)=\frac{1+\sigma}{2 \pi Y} \int d x^{\prime} d y^{\prime}\left(\frac{z^{2}}{r^{3}}+\frac{2(1-\sigma)}{r}\right) f_{z}\left(x^{\prime}, y^{\prime}\right),
$$

where $\quad r^{2} \equiv\left(x-x^{\prime}\right)^{2}+\left(y-y^{\prime}\right)^{2}+z^{2}, \quad Y \quad$ represents Young's modulus, and $\sigma$ represents Poisson's ratio of the elastic half space.

The elastic energy stored in the substrate equals the work that is done by the surface load on the deformation of the surface. As only the $z$ component of stress $\sigma_{z z}=p=f_{z}$ is different from zero, this energy reads

$$
E=\frac{1}{2} \int d x d y p(x, y) v_{z}(x, y, z=0) .
$$

We start our analysis with the surface deformation $v_{z}$ by specializing Eq. (A4) to $z=0$ :

$$
\begin{aligned}
v_{z}(x, y, 0)= & \frac{(1-\sigma)^{2}}{\pi Y} \frac{1}{(2 \pi)^{2}} \int d k_{x} d k_{y} \Phi\left(k_{x}, k_{y}\right) e^{i\left(k_{x} x+k_{y} y\right)} \\
& \times \int d x^{\prime} d y^{\prime} \frac{e^{i\left[k_{x}\left(x^{\prime}-x\right)+k_{y}\left(y^{\prime}-y\right)\right]}}{\sqrt{\left(x-x^{\prime}\right)^{2}+\left(y-y^{\prime}\right)^{2}}}
\end{aligned}
$$

Here, the two-dimensional Fourier transform of $p=f_{z}$ has been introduced as

$$
\Phi\left(k_{x}, k_{y}\right) \equiv \int d x d y p(x, y) e^{-i\left(k_{x} x+k_{y} y\right)} .
$$

Performing the integration on $x^{\prime}$ and $y^{\prime}$ in polar coordinates, we arrive at 
$v_{z}(x, y, 0)=\frac{(1-\sigma)^{2}}{\pi Y} \frac{1}{2 \pi} \int d k_{x} d k_{y} \frac{1}{k_{\perp}} \Phi\left(k_{x}, k_{y}\right) e^{i\left(k_{x} x+k_{y} y\right)}$,

where $k_{\perp}$ is definded as $k_{\perp} \equiv \sqrt{k_{x}^{2}+k_{y}^{2}}$. Inserting Eq. (A8) into Eq. (A5) and performing the integration on $x$ and $y$ results in

$$
\begin{aligned}
E= & \frac{F_{0}^{2}}{4 \pi^{3} r_{0}^{2}} \frac{(1-\sigma)^{2}}{Y} \int d k_{x} d k_{y} \frac{1}{k_{\perp}} \pi r_{0}^{2} \times\left[e^{-k_{x}^{2} r_{0}^{2} / 4}\right. \\
& \left.\times\left(e^{-k_{y}^{2} r_{0}^{2} / 4}+\frac{1}{2} e^{-\left(k_{y}+k_{s}\right)^{2} r_{0}^{2} / 4}+\frac{1}{2} e^{-\left(k_{y}-k_{s}\right)^{2} r_{0}^{2} / 4}\right)\right]^{2} .
\end{aligned}
$$

Introducing polar coordinates and performing the angular integration by applying the relation

$$
\int_{0}^{2 \pi} e^{x \cos \varphi} d \varphi=2 \pi I_{0}(x)
$$

yields

$$
\begin{aligned}
E= & \frac{F_{0}^{2}}{2 \pi} \frac{(1-\sigma)^{2}}{Y} \int d k_{\perp} \\
& \times\left\{e^{-k_{\perp}^{2} r_{0}^{2} / 2}+2 I_{0}\left(\frac{k_{s} k_{\perp} r_{0}^{2}}{2}\right) e^{-\left(2 k_{\perp}^{2}+k_{s}^{2}\right) r_{0}^{2} / 4}\right. \\
& \left.+\frac{1}{2}\left[1+I_{0}\left(k_{s} k_{\perp} r_{0}^{2}\right)\right] e^{-\left(k_{\perp}^{2}+k_{s}^{2}\right) r_{0}^{2} / 2}\right\}
\end{aligned}
$$

with $I_{0}(x)$ representing the modified Bessel function of the first kind. Replacing $k_{\perp} r_{0} / \sqrt{2} \rightarrow a$ and $k_{s} r_{0} / \sqrt{2} \rightarrow b$ and using

$$
\int_{0}^{\infty} I_{0}(c x) e^{-x^{2}} d x=\frac{\sqrt{\pi}}{2} e^{c^{2} / 8} I_{0}\left(\frac{c^{2}}{8}\right),
$$

the integration on $a$ finally yields

$$
E=\frac{F_{0}^{2}}{2 \sqrt{2 \pi}} \frac{1-\sigma^{2}}{Y r_{0}} \times G(b),
$$

with $G(b)$ as given in Eq. (26) and the prefactor coinciding with the conventional theory for a half-space by Bondu, Hello, and Vinet [13]. Thus, the function $G(b)$ actually represents the correction factor for the power noise spectrum of substrate Brownian noise considering a striped probe.

\section{APPENDIX B: THERMOELASTIC SUBSTRATE NOISE}

In the direct approach of noise calculation, the TE noise is closely connected to TE damping. The virtual force applied to the substrate causes volume dilatations, which in turn lead to the introduction of heat into parts of the substrate. Then, the heat flow between these parts can be interpreted as the loss process. To correctly describe these losses, one has to start at the elastic problem. Then, the volume dilatation $\Theta \equiv u_{x x}+u_{y y}+u_{z z}$, with the strain tensor $u_{i j}$, has to be inserted into the heat equation

$$
\partial_{t} u-a^{2} \Delta u=-\partial_{t} \frac{\alpha Y T_{0}}{\rho C_{p}(1-2 \sigma)} \Theta(x, y, z),
$$

with temperature $u$, heat capacity $C_{p}$, mean temperature $T_{0}$, mass density $\rho$, thermal conductivity $\kappa$, and heat conductivity $a^{2}=\kappa /\left(\rho C_{p}\right)$. Finally, the rate of energy loss follows

$$
P_{\mathrm{diss}}=\left\langle\int_{V} \frac{\kappa}{T_{0}}(\nabla u)^{2} d x d y d z\right\rangle,
$$

where the angle brackets account for a temporal average.

Starting at the applied force, we use Eqs. (A2), (A3), and (A4) to compute the dilatation as

$$
\begin{aligned}
\Theta(x, y, z) & =u_{x x}+u_{y y}+u_{z z} \\
& =\frac{1+\sigma}{2 \pi Y} \int d x^{\prime} d y^{\prime} \frac{2 z}{r^{3}}(2 \sigma-1) f_{z}\left(x^{\prime}, y^{\prime}\right) .
\end{aligned}
$$

In the same procedure as for Brownian substrate noise, we replace $f_{z}$ by its Fourier transform $\Phi\left(k_{x}, k_{y}\right)$ :

$$
\begin{aligned}
\Theta(x, y, z)= & \frac{(1+\sigma)(2 \sigma-1)}{\pi Y} z \int \frac{d k_{x} d k_{y}}{(2 \pi)^{2}} \Phi\left(k_{x}, k_{y}\right) e^{i\left(k_{x} x+k_{y} y\right)} \\
& \times \int d x^{\prime} d y^{\prime} \frac{e^{i\left[k_{x}\left(x^{\prime}-x\right)+k_{y}\left(y^{\prime}-y\right)\right]}}{\left[\left(x-x^{\prime}\right)^{2}+\left(y-y^{\prime}\right)^{2}+z^{2}\right]^{\{3 / 2\}}} .
\end{aligned}
$$

The integration on $x^{\prime}$ and $y^{\prime}$ is performed in polar coordinates, leading to

$$
\begin{aligned}
\Theta(x, y, z)= & \frac{(1+\sigma)(2 \sigma-1)}{2 \pi^{2} Y} \frac{z}{|z|} e^{-k_{\perp}|z|} \\
& \times \int d k_{x} d k_{y} e^{i\left(k_{x} x+k_{y} y\right)} \Phi\left(k_{x}, k_{y}\right),
\end{aligned}
$$

where again $k_{\perp}$ equals $\sqrt{k_{x}^{2}+k_{y}^{2}}$. For the sake of a simple calculation scheme, we symmetrically continue $\Theta$ to positive $z$ values. The remaining $z$ dependence in the exponential term is then transformed into Fourier space, yielding

$$
\begin{aligned}
\Theta(x, y, z)= & \frac{(1+\sigma)(2 \sigma-1)}{4 \pi^{3} Y} \int d k_{x} d k_{y} d k_{z} \\
& \times e^{i\left(k_{x} x+k_{y} y+k_{z} z\right)} \frac{2 k_{\perp}}{k_{\perp}^{2}+k_{z}^{2}} \Phi\left(k_{x}, k_{y}\right) .
\end{aligned}
$$


From this, we immediately read the Fourier transform of $\Theta$ as

$$
\Theta\left(k_{x}, k_{y}, k_{z}\right)=\frac{2(2 \sigma-1)(1+\sigma)}{Y} \frac{2 k_{\perp}}{k_{\perp}^{2}+k_{z}^{2}} \Phi\left(k_{x}, k_{y}\right) .
$$

In the next step, the heat equation [Eq. (B1)] has to be solved for the given dilatation. For this purpose, it is transformed into the Fourier space reading

$$
i \omega u(\mathbf{k})+a^{2} k^{2} u(\mathbf{k})=-i \omega A \Theta(\mathbf{k}),
$$

where the variables $\left(k_{x}, k_{y}, k_{z}\right)$ are combined into $\mathbf{k}$ and the constant $A$ equals $\alpha Y T_{0} /\left[\rho C_{p}(1-2 \sigma)\right]$. In this calculation, the Fourier transforms are defined on the full space. Further, the boundary conditions at the substrate surface, i.e., vanishing heat flow, have to be satisfied. For this purpose, mirror loads have to be applied being heat sources in our case. In a former step, $\Theta$ has been chosen as an even function in $z$ already ensuring a vanishing heat flow at the boundary.

We also have to take this point into account for the evaluation of the dissipated power using Eq. (B2). Here, an integration over all space is performed, and a subsequent division by a factor of 2 normalizes the result to the halfspace. Furthermore, also, the temporal averaging introduces a division by 2 due to the harmonic loading. Finally, we arrive at

$$
P_{\mathrm{diss}}=\frac{\kappa}{4 T_{0}} \frac{1}{(2 \pi)^{3}} \int_{\mathbb{R}^{3}} d \mathbf{k} k^{2}|u(\mathbf{k})|^{2} .
$$

Inserting Eqs. (B7) and (B8) into this expression yields

$$
P_{\text {diss }} \approx \frac{4 \kappa T_{0} \alpha^{2}(1+\sigma)^{2}}{\rho^{2} C_{p}^{2}} \int_{\mathbb{R}^{3}} \frac{d \mathbf{k}}{(2 \pi)^{3}} \frac{k_{\perp}^{2}}{k^{2}} \frac{\omega^{2} \Phi^{2}\left(k_{x}, k_{y}\right)}{\omega^{2}+a^{4} k^{4}} .
$$

Taking the approximation of small stripe periods $\left(k_{s} r_{0} \gg 1\right)$ into account, all terms $\exp \left(-k_{s}^{2} r_{0}^{2}\right)$ can be neglected in $\Phi^{2}\left(k_{x}, k_{y}\right)$, leading to

$$
\begin{aligned}
\Phi^{2}\left(k_{x}, k_{y}\right)= & F_{0}^{2} e^{-k_{x}^{2} r_{0}^{2} / 2}\left[e^{-k_{y}^{2} r_{0}^{2} / 2}\right. \\
& \left.+\frac{1}{4}\left(e^{-\left(k_{y}+k_{s}\right)^{2} r_{0}^{2} / 2}+e^{-\left(k_{y}-k_{s}\right)^{2} r_{0}^{2} / 2}\right)\right] .
\end{aligned}
$$

Examining each term in square brackets individually, starting with the first one, we find the result for a conventional Gaussian beam coinciding with the work of Braginsky, Gorodetsky, and Vyatchanin [11]. Repeating their result gives the first, coventional part of dissipation,

$$
P_{\mathrm{diss}}^{(1)}=\frac{4 \kappa T_{0} \alpha^{2}(1+\sigma)^{2}}{\rho^{2} C_{p}^{2}} \frac{F_{0}^{2}}{(2 \pi)^{2}}\left(\frac{\sqrt{\omega}}{a}\right)^{3} \mathscr{I}(b),
$$

where $b$ is defined as $\omega r_{0}^{2} /\left(2 a^{2}\right)$ and

$$
\mathscr{I}(b) \equiv \int_{-1}^{1} d q \int_{0}^{\infty} d y\left(1-q^{2}\right) \frac{y^{2}}{1+y^{4}} e^{-\left(1-q^{2}\right) y^{2} b} .
$$

The second term in square brackets of Eq. (B11) is caused by the stripe pattern and increases the conventional noise value. Performing the integration on $k_{y}$ of this part, we assume that the exponentials are falling so strongly that outside the exponent $k_{y}$ can be replaced by the stripe's wave number $k_{s}$. This approximation is especially fulfilled if the factor $a^{2} / \omega$ representing the thermal path length is small compared to the beam radius $r_{0}$. In the same way, the integration on $k_{x}$ is performed, yielding

$$
\begin{aligned}
P_{\mathrm{diss}}^{(2)}= & \frac{\kappa T_{0} \alpha^{2}(1+\sigma)^{2}}{\rho^{2} C_{p}^{2}} F_{0}^{2} \frac{1}{2 \pi^{2}} \frac{k_{s}}{r_{0}^{2}} \\
& \times \int_{\mathbb{R}^{3}} d\left(\frac{k_{z}}{k_{s}}\right) \frac{1}{1+\frac{k_{z}^{2}}{k_{s}^{2}}} \frac{1}{1+\frac{a^{4} k_{s}^{4}}{\omega^{2}}\left(1+\frac{k_{z}^{2}}{k_{s}^{2}}\right)^{2}} .
\end{aligned}
$$

This last integral is solved as

$$
P_{\mathrm{diss}}^{(2)}=\frac{1}{2 \pi} \frac{\kappa \alpha^{2}(1+\sigma)^{2}}{\rho^{2} C_{p}^{2}} \frac{k_{s}}{r_{0}^{2}} T_{0} F_{0}^{2} \times\left(1-\frac{\sqrt{1+\zeta}}{\sqrt{2} \zeta}\right),
$$

where $\zeta$ is defined as

$$
\zeta \equiv \sqrt{1+\frac{\omega^{2}}{a^{4} k_{s}^{4}}} .
$$

The interesting case for GEO 600 can be found in the limit of a thermal path length being small compared to the stripe period $\left(k_{s}^{2} a^{2} / \omega \ll 1\right)$. In this limit, $\zeta$ becomes very large, leading to the following simplification of Eq. (B15):

$$
P_{\mathrm{diss}}^{(2)} \simeq \frac{1}{2 \pi} \frac{\kappa \alpha^{2}(1+\sigma)^{2}}{\rho^{2} C_{p}^{2}} \frac{k_{s}}{r_{0}^{2}} T_{0} F_{0}^{2} .
$$

\section{APPENDIX C: THERMOELASTIC COATING NOISE}

In typical test masses, the coating stack is small compared to the substrate dimensions. Thus, the response of the mirror on the virtual load is nearly fully determined by the substrate. In the following, we use this substrate response to obtain the elastic fields in the coating via transition conditions applying the idea of Harry et al. [12]. For TE coating noise, only the heat insertion due to the dilatation of the coating is taken into account in the thermal 
analysis, i.e., the calculation of the dissipated power. In the following, we apply this heat as a thin surface load neglecting the coating thickness. Further, only the heat flow in the substrate is considered. This simplification can lead to a deviation from the correct result, especially at high frequencies and for thick coatings. Nevertheless, this model is used here due to its analytical clarity and serves as an exemplary system to determine the effect of stripes on this kind of noise.

Again, we start at the deformation of the surface of the half-space given in Eqs. (A2), (A3), and (A4). From these quantities, one can calculate the following sum of strain components:

$u_{x x}+u_{y y}=\frac{1+\sigma}{2 \pi Y} \int d x^{\prime} d y^{\prime} f_{z}\left(x^{\prime}, y^{\prime}\right) \frac{z}{r^{5}}\left[r^{2}(2 \sigma-2)+3 z^{2}\right]$.

Using the same technique as presented for TE substrate noise (see Appendix B), we find

$$
\begin{aligned}
u_{x x}+u_{y y}= & \frac{1+\sigma}{(2 \pi)^{2} Y} \times \int d k_{x} d k_{y} e^{i\left(k_{x} x+k_{y} y\right)} \Phi\left(k_{x}, k_{y}\right) \\
& \times e^{-k_{\perp}|z|}\left[(2 \sigma-2) \frac{z}{|z|}+\frac{z}{|z|}\left(1+k_{\perp}|z|\right)\right]
\end{aligned}
$$

by using the two-dimensional Fourier transform $\Phi\left(k_{x}, k_{y}\right)$ of the virtual load $f_{z}\left(x^{\prime}, y^{\prime}\right)$. Compared to Eq. (B5), the second summand originates from the $1 / r^{5}$ term of Eq. (C1).

With the above result, we can obtain an expression for the volume dilatation in the coating $\Theta_{c}$ as the crucial term for heat sources in the coating. This term is defined by the trace of the strain tensor in the coating

$$
\Theta_{c}=u_{x x, c}+u_{y y, c}+u_{z z, c} .
$$

Because of the transition conditions, the components $u_{x x}$, $u_{y y}$, and $\sigma_{z z}$ are steady at the substrate-coating boundary. Using the basic equations of elasticity [30] for the isotropic coating, $u_{z z, c}$ can be replaced by the steady components, yielding

$$
\Theta_{c}(z=0)=\frac{1-2 \sigma_{c}}{1-\sigma_{c}}\left[\frac{1+\sigma_{c}}{Y_{c}} \sigma_{z z}+\left(u_{x x}+u_{y y}\right)\right],
$$

where $Y_{c}$ and $\sigma_{c}$ represent Young's modulus and Poisson's ratio for the coating layer. In the last equations, all elastic field components of the coating have been replaced by the fields at the substrate's surface due to the transition conditions. As we assume the probing force acting at the coating-air surface, the coating's stress component $\sigma_{z z}$ is given by the virtual load $f_{z}$. But the elastic solution of Landau and Lifshitz [30] is valid for a half-space lying in the positive $z$ axis. As the stress is defined with respect to the surface normal (pointing along the negative $z$ direction), a minus sign has to be introduced, leading to

$$
\begin{aligned}
\Theta_{c}(z=0)= & \frac{1}{(2 \pi)^{2}} \int d k_{x} d k_{y} \Phi\left(k_{x}, k_{y}\right) e^{i\left(k_{x} x+k_{y} y\right)} \\
& \times \frac{1-2 \sigma_{c}}{1-\sigma_{c}}\left[-\frac{1+\sigma_{c}}{Y_{c}}+\frac{1+\sigma}{Y}(2 \sigma-1)\right] .
\end{aligned}
$$

Assuming a small height $H$ of the coating layer, the $z$ dependence on $\Theta_{c}$ is modelled as a delta distribution $\Theta_{c}(x, y, z)=\Theta_{c}(x, y, 0) H \delta(z)$. The three-dimensional Fourier transform of $\Theta_{c}$ then reads

$$
\Theta_{c}\left(k_{x}, k_{y}, k_{z}\right)=H \Phi\left(k_{x}, k_{y}\right)\left(1-2 \sigma_{c}\right) M,
$$

with $M$ defined as

$$
M \equiv \frac{1}{1-\sigma_{c}}\left[-\frac{1+\sigma_{c}}{Y_{c}}+\frac{1+\sigma}{Y}(2 \sigma-1)\right]
$$

Please note that $M$ simplifies to $-2(1+\sigma) / Y$ for the case of identical substrate and coating materials $\left(Y=Y_{c}\right.$ and $\sigma=\sigma_{c}$ ).

In analogy to Appendix B, the heat equation is solved in the Fourier space. The only modification occurs in the consideration of mirror heat sources. As, again, the whole space is considered, the surface loads at the boundary have simply to be doubled in their amplitude. Using Eq. (B2), we end up at a dissipated power of

$$
P_{\mathrm{diss}}=\frac{\kappa \alpha^{2} Y_{c}^{2} M^{2} H^{2} T_{0}}{(2 \pi)^{3} \rho^{2} C_{p}^{2}} \int_{\mathbb{R}^{3}} d \mathbf{k} \frac{k^{2}}{1+\frac{a^{4}}{\omega^{2}} k^{4}} \Phi^{2}\left(k_{x}, k_{y}\right) .
$$

In the following, we use the approximation of $\Phi^{2}\left(k_{x}, k_{y}\right)$ for small stripe periods presented in Eq. (B11). Performing the same approximative integration on $k_{x}$ and $k_{y}$ as in Appendix B, i.e., for thermal path lengths small compared to the beam radius, one finds for the first term in brackets:

$$
P_{\mathrm{diss}}^{(1)}=\frac{\sqrt{2}}{8 \pi} \frac{\alpha^{2} Y_{c}^{2} M^{2} H^{2} T_{0}}{r_{0}^{2} \sqrt{\kappa \rho C_{p}}} F_{0}^{2} \omega^{3 / 2}
$$

Specialized to substrate and coating materials exhibiting identical elastic properties, this expression coincides with the result of Braginsky and Vyatchanin [15] for a conventional Gaussian beam.

The effect due to stripes shows up in the remaining terms of $\Phi^{2}$ in Eq. (C8). Performing the same integration scheme on $k_{x}$ and $k_{y}$ as in Appendix B leads to 


$$
\begin{aligned}
P_{\text {diss }}^{(2)}= & \frac{\kappa \alpha^{2} Y_{c}^{2} M^{2} H^{2} T_{0}}{16 \pi^{2} r_{0}^{2} \rho^{2} C_{p}^{2}} F_{0}^{2} \\
& \times k_{s}^{3} \int_{\mathbb{R}} d\left(\frac{k_{z}}{k_{s}}\right) \frac{2 \times\left[1+\left(\frac{k_{z}}{k_{s}}\right)^{2}\right]}{1+\frac{a^{4}}{\omega^{2}} k_{s}^{4}\left[1+\left(\frac{k_{z}}{k_{s}}\right)^{2}\right]^{2}} .
\end{aligned}
$$

Solving this integral yields

$$
P_{\text {diss }}^{(2)}=\frac{\alpha^{2} Y_{c}^{2} M^{2} H^{2} T_{0}}{8 \pi r_{0}^{2} \kappa k_{s}} \omega^{2} F_{0}^{2} \times \sqrt{\frac{1+\zeta}{2 \zeta^{2}}},
$$

with

$$
\zeta=\sqrt{1+\frac{\omega^{2}}{a^{4} k_{s}^{4}}}
$$

For the thermal path length small compared to the stripe period $\left(a^{4} / \omega^{2} \ll 1 / k_{s}^{4}\right)$, the result is well approximated by

$$
P_{\text {diss }}^{(2)} \approx \frac{\sqrt{2}}{16 \pi} \frac{\alpha^{2} Y_{c}^{2} M^{2} H^{2} T_{0}}{r_{0}^{2} \sqrt{\kappa \rho C_{p}}} F_{0}^{2} \omega^{3 / 2} .
$$

In this limit, no dependence on the stripe period is observed. Such a behavior is well understood through rough qualitative considerations. We begin this argument by looking at a sphere exhibiting the thermal path length as diameter. Such a sphere is illustrated in Fig. 10. Within this sphere, no significant temperature gradient can build up, and thus its temperature can be treated to be homogeneous. Consequently, a significant temperature fluctuation can only happen outside this sphere. Using a basic equation of thermodynamics, the temperature fluctuation of this sphere is given by its volume and follows

$$
\left(\Delta T_{s}\right)^{2}=\frac{k_{B} T^{2}}{\rho C_{p} V} \simeq \frac{k_{B} T^{2}}{\rho C_{p} r_{\text {th }}^{3}} .
$$

By means of the coefficient of thermal expansion $\alpha$, this temperature change leads to a deformation of the substrate surface of

$$
\Delta z_{s} \simeq \alpha r_{\text {th }} \Delta T_{s},
$$

due to a single sphere. Here, we focus on the TE noise of the coating. With its thickness $H$, the coating is assumed to be smaller than the thermal path length. Thus, the deformation due to temperature fluctuations in the coating is restricted to a height $H$, leading to the replacement $r_{\text {th }} \rightarrow H$ in Eq. (C15).

Finally, the surface deformation caused by temperature fluctuations within the coating can be averaged by the independent effect of $N$ such spheres. As the thickness of the coating $H$ is assumed to be small compared to the radius

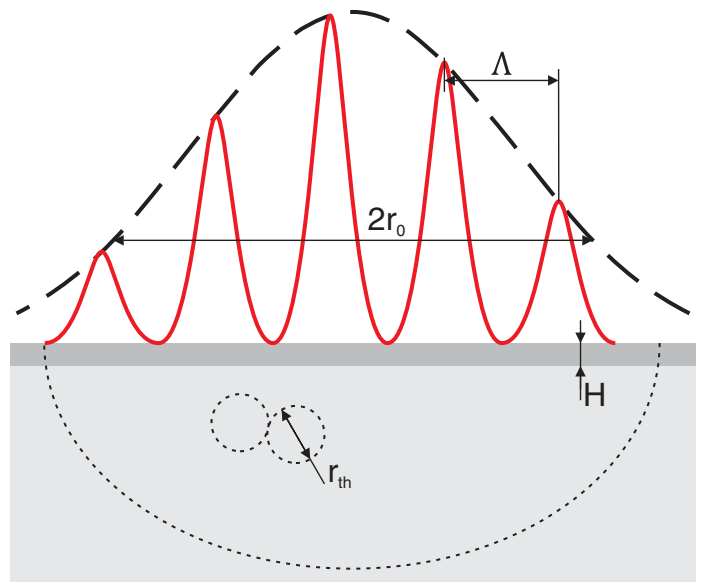

FIG. 10 (color online). Sketch for a qualitative explanation of TE coating noise for the limiting case of small thermal path lengths $r_{\text {th }}$. In this approach, the sample is divided into spheres showing a radius of the thermal path length $r_{\text {th }}$. Within them, the temperature is nearly constant, and temperature fluctuations in two spheres are nearly independent. This scheme allows the correct derivation of the thermal noise level with respect to general dependencies on physical parameters.

of the spheres $r_{\text {th }}$, their number is given by a segmentation of the substrate surface on the beam spot. This approach yields $N \simeq r_{0}^{2} / r_{\text {th }}^{2}$. The sum of $N$ independent but equally distributed noise sources is then given by

$$
\Delta z=\frac{\Delta z_{s}}{\sqrt{N}} \simeq \alpha H \sqrt{\frac{k_{B} T^{2}}{\omega \kappa \rho C_{p} r_{0}^{2}}} .
$$

This simple estimate shows the same dependencies as the exact result given in Eq. (29) with only a slight deviation in the prefactor.

\section{APPENDIX D: THERMOREFRACTIVE COATING NOISE}

In this work, we are mainly interested in the TR coating noise of highly reflective multilayer coatings. Further, we assume that the coating is thin compared to its thermal path length. Then, one can assume the whole layer stack to show the same temperature. A fluctuation is then only produced by a homogeneous temperature change of the whole structure. Following the direct noise calculation scheme for TR noise [9], the phase change in the reflected light due to a temperature change $\Delta T$ is to be considered. Following Refs. [16,17] for a typical quarter-wavelength geometry exhibiting more than six layer pairs, this phase change reads

$$
\Delta \varphi \approx \frac{4 \pi}{\lambda} \times \beta_{\mathrm{eff}} \lambda \Delta T,
$$

where $\beta_{\text {eff }}$ is defined in Eq. (31). 
In the second step, a virtual heat source is to be introduced into the coating. Assuming an infinitely thin heat source in the $z$ direction, we are only interested in the total integral of this heat source along the $z$ direction. This heat term follows

$$
q(x, y)=\beta_{\mathrm{eff}} \lambda T_{0} \times p(x, y) e^{i \omega t},
$$

where $p$ represents the probing force at the coating. The temperature field due to this heat term is governed by the heat equation

$$
\partial_{t} u-a^{2} \Delta u=\frac{\partial_{t} q}{\rho C_{p}} .
$$

This equation exhibits the close analogy to the calculation of TE substrate noise. By examing Eqs. (B1) and (C7), the only modification is found in the replacement

$$
\alpha Y_{c} H M \rightarrow \beta_{\text {eff }} \lambda
$$

to adapt TE coating noise to the case of TR coating noise. With this rule, the dissipated power follows

$$
P_{\mathrm{diss}}=\frac{\kappa}{T_{0}} \int_{\mathbb{R}^{3}} \frac{d \mathbf{k}}{(2 \pi)^{3}} k^{2} \frac{\omega^{2}}{\omega^{2}+a^{4} k^{4}} \frac{\beta_{\mathrm{eff}}^{2} \lambda^{2} T_{0}^{2}}{\rho^{2} C_{p}^{2}} \Phi^{2}\left(k_{x}, k_{y}\right),
$$

with $\Phi\left(k_{x}, k_{y}\right)$ representing the Fourier transform of $p(x, y)$. From this point, the same considerations as in Appendix $\mathrm{C}$ hold leading to the result of the conventional case of

$$
P_{\mathrm{diss}}^{(1)}=\frac{\sqrt{2}}{8 \pi} \frac{\beta_{\mathrm{eff}}^{2} \lambda^{2} T_{0}}{r_{0}^{2} \sqrt{\kappa \rho C_{p}}} F_{0}^{2} \omega^{3 / 2}
$$

The additional contribution due to the striped probe pattern follows in the same way as

$$
P_{\mathrm{diss}}^{(2)}=\frac{\beta_{\mathrm{eff}}^{2} \lambda^{2} T_{0}}{8 \pi r_{0}^{2} \kappa k_{s}} \omega^{2} F_{0}^{2} \times \sqrt{\frac{1+\zeta}{2 \zeta^{2}}},
$$

where $\zeta$ is given in Eq. (C12). In the case of a thermal path length small compared to the stripe period, this expression can be further simplified to

$$
P_{\mathrm{diss}}^{(2)}=\frac{\sqrt{2}}{16 \pi} \frac{\beta_{\mathrm{eff}}^{2} \lambda^{2} T_{0}}{r_{0}^{2} \sqrt{\kappa \rho C_{p}}} F_{0}^{2} \omega^{3 / 2},
$$

again being independent from the stripe period.

\section{APPENDIX E: BROWNIAN COATING NOISE}

Using the model of structural damping for Brownian noise, the dissipated power can be calculated with the help of the strain energy amplitude $E_{\max }$ in the coating via

$$
P_{\mathrm{diss}}=\omega \phi E_{\max }
$$

where $\phi$ represents the mechanical loss of the coating material. Thus, in the following, we calculate the strain energy in a coating layer on top of an elastic half-space. Again, due to the small coating thickness, we use the substrate solution to the external force neglecting the coating. The coating elastic fields are then derived via transition conditions. In the following, we divide the strain energy density $w=w_{\mathrm{n}}+w_{\mathrm{sh}}$ into normal $\left(w_{\mathrm{n}}\right)$ and shear $\left(w_{\text {sh }}\right)$ contributions via

$$
\begin{gathered}
w_{\mathrm{n}}=\frac{1}{2}\left(u_{x x} \sigma_{x x}+u_{y y} \sigma_{y y}+u_{z z} \sigma_{z z}\right) \\
w_{\mathrm{sh}}=u_{x y} \sigma_{x y}+u_{x z} \sigma_{x z}+u_{y z} \sigma_{y z} .
\end{gathered}
$$

To obtain the coating energy, the elastic fields of the coating have to be inserted into the terms above. For a direct application of the substrate solution, the equations of elasticity are used to rephrase the coating energy in terms of fields being continuous at the boundary. For the normal energy, this expression reads

$$
\begin{aligned}
w_{\mathrm{n}}= & \frac{\left(1+\sigma_{c}\right)\left(1-2 \sigma_{c}\right)}{2 Y_{c}\left(1-\sigma_{c}\right)} \sigma_{z z}^{2}+\frac{Y_{c}}{4} \frac{\left(u_{x x}+u_{y y}\right)^{2}}{1-\sigma_{c}} \\
& +\frac{Y_{c}}{4} \frac{\left(u_{x x}-u_{y y}\right)^{2}}{1+\sigma_{c}} .
\end{aligned}
$$

As the virtual force is exclusively acting along the $z$ direction, the stress components $\sigma_{x z}$ and $\sigma_{y z}$ have to vanish at the coating air boundary. Because of the small coating thickness, they also vanish in the whole coating. Consequently, the shear energy simplifies to

$$
w_{\mathrm{sh}}=\frac{Y_{c}}{1+\sigma_{c}} u_{x y}^{2} \text {. }
$$

The total elastic energy of the coating is obtained by an integration of $w$ on the coating volume. Because of the small coating thickness $H$, the elastic fields in the coating are assumed to be constant along the $z$ axis, yielding

$$
E_{\max }=\int_{0}^{H} w d x d y d z=H \int w d x d y .
$$

Thus, in the following, we present the integrations on the four combinations of elastic fields in $w$. 
1st integral, $\propto \sigma_{z z}^{2}$ The term on $\sigma_{z z}$ is easily calculated directly:

$$
\begin{aligned}
\int d x d y \sigma_{z z}^{2} & =\int d x d y \frac{F_{0}^{2}}{\pi^{2} r_{0}^{4}} e^{-2\left(x^{2}+y^{2}\right) / r_{0}^{2}}\left[1+\cos \left(k_{s} y\right)\right]^{2} \\
& \approx \frac{F_{0}^{2}}{\pi^{2} r_{0}^{2}} \frac{\pi r_{0}^{2}}{2}\left(1+0+\frac{1}{2}\right)=\frac{3}{4} \frac{F_{0}^{2}}{\pi r_{0}^{2}} .
\end{aligned}
$$

Here, again, the approximation of small stripe periods $k_{s}^{2} r_{0}^{2} \gg 1$ has been used, and respective exponential terms have been neglected. The first term in brackets corresponds to the conventional case, while the last two occur due to the striped readout exhibiting an increase of $50 \%$.

2nd integral, $\propto\left(u_{x x}+u_{y y}\right)^{2}$ Inspecting Eq. (C2) at $z=0$ easily yields the two-dimensional Fourier spectrum of $u_{x x}+u_{y y}$ as

$$
u_{x x}+u_{y y}=-\frac{(1+\sigma)(1-2 \sigma)}{Y} \Phi\left(k_{x}, k_{y}\right) .
$$

Using Parseval's theorem, the desired integral on $x$ and $y$ can be expressed by the Fourier transform as

$$
\begin{aligned}
& \int d x d y\left(u_{x x}+u_{y y}\right)^{2}=\frac{1}{(2 \pi)^{2}} \int d k_{x} d k_{y}\left(u_{x x}+u_{y y}\right)^{2} \\
& =\frac{(1+\sigma)^{2}(1-2 \sigma)^{2}}{(2 \pi Y)^{2}} \int d k_{x} d k_{y} \Phi^{2}\left(k_{x}, k_{y}\right) .
\end{aligned}
$$

Inserting $\Phi^{2}\left(k_{x}, k_{y}\right)$ from Eq. (B11) yields

$$
\int d x d y\left(u_{x x}+u_{y y}\right)^{2}=\frac{3}{4} \frac{F_{0}^{2}}{\pi r_{0}^{2}} \frac{(1+\sigma)^{2}(1-2 \sigma)^{2}}{Y^{2}} .
$$

In analogy to the $\sigma_{z z}$ term, the conventional case gives a prefactor of $1 / 2$, and the striped readout introduces an additional increase by $50 \%$.

$3 r d$ integral, $\propto u_{x y}^{2}$ Beginning with the formal solution for the shear strain and using Eqs. (A2) and (A3) yields

$$
\begin{aligned}
u_{x y}= & \frac{1}{2}\left(\partial_{y} v_{x}+\partial_{x} v_{y}\right) \\
= & \frac{1+\sigma}{2 \pi Y} \int d x^{\prime} d y^{\prime}\left(x-x^{\prime}\right)\left(y-y^{\prime}\right) f_{z}\left(x^{\prime}, y^{\prime}\right) \\
& \times\left[-\frac{3 z}{r^{5}}+\frac{1-2 \sigma}{r^{2}(z+r)^{2}}+\frac{1-2 \sigma}{r^{3}(z+r)}\right] .
\end{aligned}
$$

Evaluating this expression at the surface, i.e., at $z=0$, largely simplifies the equation. Further, introducing the Fourier transform of $f_{z}$ and two variable transforms $\left(x^{\prime}-x \rightarrow x^{\prime}\right.$ and $\left.y^{\prime}-y \rightarrow y^{\prime}\right)$, we find the Fourier transform $u_{x y}\left(k_{x}, k_{y}\right)$ as

$$
\begin{aligned}
u_{x y}\left(k_{x}, k_{y}\right)= & \frac{(1-2 \sigma)(1+\sigma)}{\pi Y} \Phi\left(k_{x}, k_{y}\right) \\
& \times \int d x^{\prime} d y^{\prime} x^{\prime} y^{\prime} \frac{e^{i\left(k_{x} x^{\prime}+k_{y} y^{\prime}\right)}}{\left(x^{\prime 2}+y^{\prime 2}\right)^{2}} .
\end{aligned}
$$

Introducing polar coordinates $(\rho, \varphi)$ changes the integral to

$$
\int d \rho d \varphi \rho \frac{\rho^{2} \sin (2 \varphi)}{2 \rho^{4}} e^{i k_{\perp} \rho \cos \left(\varphi-\varphi_{k}\right)}
$$

with $k_{\perp} \equiv \sqrt{k_{x}^{2}+k_{y}^{2}}$ and $\tan \varphi_{k} \equiv k_{y} / k_{x}$. The integration on $\varphi$ is solved with the help of the following integrals:

$$
\begin{gathered}
\int_{0}^{2 \pi} d \varphi e^{i a \cos \varphi} \cos (2 \varphi)=-2 \pi J_{2}(|a|), \\
\int_{0}^{2 \pi} d \varphi e^{i a \cos \varphi} \sin (2 \varphi)=0 .
\end{gathered}
$$

Performing the coordinate transform $\varphi-\varphi_{k} \rightarrow \varphi$ and using the addition theorem for the sine function on Eq. (E14) finally yields

$$
-\pi \sin \left(2 \varphi_{k}\right) \int_{0}^{\infty} d \rho \frac{1}{\rho} J_{2}\left(\left|k_{\perp} \rho\right|\right)=-\frac{\pi}{2} \sin \left(2 \varphi_{k}\right) .
$$

Inserting the Fourier transform of $u_{x y}$ into Parseval's theorem gives the desired integral. Using the relation $\sin (2 \arctan x)=2 x /\left(1+x^{2}\right)$ gives

$$
\int d x d y u_{x y}^{2}=\frac{(1+\sigma)^{2}(1-2 \sigma)^{2}}{(2 \pi Y)^{2}} \int d k_{x} d k_{y} \frac{k_{x}^{2} k_{y}^{2} \Phi^{2}\left(k_{x}, k_{y}\right)}{\left(k_{x}^{2}+k_{y}^{2}\right)^{2}} .
$$

Inserting the first term of $\Phi^{2}$ from Eq. (B11), the conventional term is evaluated via polar coordinates as

$$
\left.\int d x d y u_{x y}^{2}\right|_{(1)}=\frac{1}{16} \frac{F_{0}^{2}}{\pi r_{0}^{2}} \frac{(1+\sigma)^{2}(1-2 \sigma)^{2}}{Y^{2}} .
$$

Considering the remaining two terms of Eq. (B11) yields the effect with respect to the striped readout. The integrals on $k_{x}$ and $k_{y}$ from Eq. (E18) are performed in polar coordinates and yield

$$
\begin{aligned}
& F_{0}^{2} \int d k_{\perp} d \varphi k_{\perp} \frac{\sin ^{2}(2 \varphi)}{4} e^{-k_{\perp}^{2} r_{0}^{2} / 2} \frac{e^{-k_{s}^{2} r_{0}^{2} / 2}}{4} \\
& \quad \times\left(e^{k_{\perp} k_{s} r_{0}^{2} \sin \varphi}+e^{-k_{\perp} k_{s} r_{0}^{2} \sin \varphi}\right) .
\end{aligned}
$$

Integrating on $\varphi$ and subsequently on $k_{\perp}$ yields 


$$
\pi \frac{k_{s}^{2} r_{0}^{2}-3}{k_{s}^{4} r_{0}^{6}}+\pi \frac{e^{-k_{s}^{2} r_{0}^{2} / 2}}{2}\left(6+k_{s}^{2} r_{0}^{2}\right) .
$$

For the approximation of small stripe periods $k_{s} r_{0} \gg 1$ in leading order, we find

$$
\left.\int d x d y u_{x y}^{2}\right|_{(2)} \approx \frac{1}{4} \frac{1}{k_{s}^{2} r_{0}^{2}} \frac{F_{0}^{2}}{\pi r_{0}^{2}} \frac{(1+\sigma)^{2}(1-2 \sigma)^{2}}{Y^{2}} .
$$

Thus, the contribution of the striped readout is of higher order in $k_{s} r_{0}$ than the conventional term. For the approximation of a small stripe period $k_{s} r_{0} \gg 1$, the effect of the modified readout is negligibly small for this third integral.

4th integral $\propto\left(u_{x x}-u_{y y}\right)^{2}$ This last term can be solved by the same methods as presented for the third integral with minor modifications. For this reason, we only state the main results here. Using the basic elastic equations of the half-space and the Fourier calculus, we find the following expression for the integral:

$$
\begin{aligned}
\int d x d y\left(u_{x x}-u_{y y}\right)^{2}= & \frac{(1+\sigma)^{2}(1-2 \sigma)^{2}}{(2 \pi Y)^{2}} \\
& \times \int d k_{x} d k_{y}\left(\frac{k_{x}^{2}-k_{y}^{2}}{k_{x}^{2}+k_{y}^{2}}\right)^{2} \Phi^{2}\left(k_{x}, k_{y}\right) .
\end{aligned}
$$

The conventional part then reads

$$
\left.\int d x d y\left(u_{x x}-u_{y y}\right)^{2}\right|_{(1)}=\frac{1}{4} \frac{F_{0}^{2}}{\pi r_{0}^{2}} \frac{(1+\sigma)^{2}(1-2 \sigma)^{2}}{Y^{2}} .
$$

In contrast to the third integral, the nonconventional contribution enters the result in the approximation of small stripe periods. In this approximation, the fourth integral reads

$$
\left.\int d x d y\left(u_{x x}-u_{y y}\right)^{2}\right|_{(2)}=\frac{1}{4} \frac{F_{0}^{2}}{\pi r_{0}^{2}} \frac{(1+\sigma)^{2}(1-2 \sigma)^{2}}{Y^{2}},
$$

showing an increase by $100 \%$ compared to the conventional case.
Inserting the above four integrals into the expression for the strain energy density allows the evaluation of the energy stored in the coating, the dissipated power, and finally the Brownian coating noise. For the conventional case, we find a total coating energy of

$$
\begin{aligned}
E_{\max }= & \frac{H F_{0}^{2}}{4 \pi r_{0}^{2}}\left[\frac{1}{Y_{c}} \frac{\left(1+\sigma_{c}\right)\left(1-2 \sigma_{c}\right)}{1-\sigma_{c}}\right. \\
& \left.+\frac{Y_{c}}{Y^{2}} \frac{(1+\sigma)^{2}(1-2 \sigma)^{2}}{1-\sigma_{c}^{2}}\right],
\end{aligned}
$$

revealing a complete coincidence with the conventional result of Harry et al. [12]. Further, the change in coating energy due to a change to the striped readout has been evaluated in the approximation of small stripe periods $\left(k_{s} r_{0} \gg 1\right)$. Here, we find an increase of the energy stored in the coating by 50\% independent from any coating or substrate parameters. Thus, the simple intuitive model from Sec. IV B is fully validated by the above analytical solution.

In the above calculation, only the term on $\sigma_{z z}$ does not depend on the substrate properties. Thus, considering finite substrate sizes, this contribution is expected to be unaltered in contrast to the remaining three terms. This assumption has been successfully observed in a finite element analysis. Further, the effect of finite test masses has been validated to produce a slightly reduced noise increase by only $45 \%$ for tantala and $49 \%$ for silica at the GEO 600 geometry. It confirms our theory and shows deviations due to finite size effects to be negligibly small in GEO 600 .

\section{APPENDIX F: DEFINITION OF FOURIER TRANSFORM}

For many steps in the above derivations, the Fourier transform is crucial. To facilitate the confirmability of these calculations, we shortly sketch our definition of the Fourier transform. Within this work, a function $u(\mathbf{r}, t)$ and its Fourier transform $u(\mathbf{k}, t)$ is given by

$$
\begin{array}{r}
u(\mathbf{k}, \omega)=\int u(\mathbf{r}, t) e^{-i(\omega t+\mathbf{k r})} d \mathbf{r} d t, \\
u(\mathbf{r}, t)=\frac{1}{(2 \pi)^{4}} \int u(\mathbf{k}, \omega) e^{i(\omega t+\mathbf{k r})} d \mathbf{k} d \omega .
\end{array}
$$

[1] P. R. Saulson, Fundamentals of Interferometric Gravitational Wave Detectors (World Scientific, Singapore, 1994).

[2] B. Abbott et al., Rep. Prog. Phys. 72, 076901 (2009).

[3] T. Accadia et al., JINST 7, P03012 (2012).
[4] H. Grote, Classical Quantum Gravity 27, 084003 (2010).

[5] B. J. Meers, Phys. Rev. D 38, 2317 (1988).

[6] R. Nawrodt, S. Rowan, J. Hough, M. Punturo, F. Ricci, and J.-Y. Vinet, Gen. Relativ. Gravit. 43, 593 (2011). 
[7] B. Benthem and Y. Levin, Phys. Rev. D 80, 062004 (2009).

[8] Y. Levin, Phys. Rev. D 57, 659 (1998).

[9] Y. Levin, Phys. Lett. A 372, 1941 (2008).

[10] A. Gillespie and F. Raab, Phys. Rev. D 52, 577 (1995).

[11] V. B. Braginsky, M. L. Gorodetsky, and S. P. Vyatchanin, Phys. Lett. A 264, 1 (1999).

[12] G. M. Harry, A. M. Gretarsson, P. R. Saulson, S. E. Kittelberger, S. D. Penn, W. Startin, S. Rowan, M. Fejer, D. R. M. Crooks, G. Cagnoli, J. Hough, and N. Nakagawa, Classical Quantum Gravity 19, 897 (2002).

[13] F. Bondu, P. Hello, and J.-Y. Vinet, Phys. Lett. A 246, 227 (1998).

[14] J.-Y. Vinet, Living Rev. Relativity 12, 5 (2009), Online Article: cited 20.01.2014.

[15] V. B. Braginsky and S. P. Vyatchanin, Phys. Lett. A 312, 244 (2003).

[16] V. B. Braginsky, M. L. Gorodetsky, and S. P. Vyatchanin, Phys. Lett. A 271, 303 (2000).

[17] M. Evans, S. Ballmer, M. Fejer, P. Fritschel, G. Harry, and G. Ogin, Phys. Rev. D 78, 102003 (2008).

[18] M. M. Fejer, S. Rowan, G. Cagnoli, D. R. M. Crooks, A. Gretarsson, G. M. Harry, J. Hough, S. D. Penn, P. H. Sneddon, and S. P. Vyatchanin, Phys. Rev. D 70, 082003 (2004).

[19] S. D. Penn, A. Ageev, D. Busby, G. M. Harry, A. M. Gretarsson, K. Numata, and P. Willems, Phys. Lett. A 352, 3 (2006).
[20] S. D. Penn, P. H. Sneddon, H. Armandula, J. C. Betzwieser, G. Cagnoli, J. Camp, D. R. M. Crooks, M. M. Fejer, A. M. Gretarsson, G. M. Harry, J. Hough, S. E. Kittelberger, M. J. Mortonson, R. Route, S. Rowan, and C. C. Vassiliou, Classical Quantum Gravity 20, 2917 (2003).

[21] R. Flaminio, J. Franc, C. Michel, N. Morgado, L. Pinard, and B. Sassolas, Classical Quantum Gravity 27, 084030 (2010).

[22] J. Franc, N. Morgado, R. Flaminio, R. Nawrodt, I. Martin, L. Cunningham, A. Cumming, S. Rowan, and J. Hough, ET-021-09, 2009, available at http://www.et-gw.eu/.

[23] Y. T. Liu and K. S. Thorne, Phys. Rev. D 62, 122002 (2000).

[24] K. Somiya and K. Yamamoto, Phys. Rev. D 79, 102004 (2009).

[25] M. L. Gorodetsky, Phys. Lett. A 372, 6813 (2008).

[26] H. Grote, K. Danzmann, K. L. Dooley, R. Schnabel, J. Slutsky, and H. Vahlbruch, Phys. Rev. Lett. 110, 181101 (2013).

[27] M. Gorodetsky and I. Grudinin, J. Opt. Soc. Am. B 21, 697 (2004).

[28] A. Chijioke, Q.-F. Chen, A. Y. Nevsky, and S. Schiller, Phys. Rev. A 85, 053814 (2012).

[29] S. W. Ballmer and D. J. Ottaway, Phys. Rev. D 88, 062004 (2013).

[30] L. D. Landau and E. M. Lifshitz, Theory of Elasticity (Pergamon Press, New York, 1986). 